\title{
Theory of the magnetization and exchange-enhanced susceptibility of alloys. I. Zero-temperature susceptibility of paramagnetic alloys in the random-phase approximation
}

\author{
Nicholas Kioussis* and J. W. Garland \\ Department of Physics, University of Illinois at Chicago, Chicago, Illinois 60680
}

A. Gonis

Division of Chemistry and Materials Science, L-280, Lawrence Livermore National Laboratory, Livermore, California 94550

(Received 8 December 1986)

\begin{abstract}
A new theoretical treatment of the magnetic susceptibility of substitutionally disordered alloys is presented. This treatment goes beyond the existing treatments of the susceptibility of alloys in several important respects, although in the present paper (I of this series) it is confined to zero temperature and the random-phase approximation (RPA). Unlike treatments based on the singlesite approximation, it yields results for the exchange-enhanced local susceptibilities $\chi_{i j}(\omega)$ as a function of the local environment of sites $i$ and $j$. Specifically, it includes the strong effects of the nonlinearity of the dependence of the $\chi_{i j}(\omega)$ on local environments and the effects of short-range order on the uniform susceptibility $\chi(0, \omega)$. This treatment goes beyond all previous cluster treatments of the susceptibility by treating exactly the embedding of a cluster in a given effective medium. Even more important, it is the first cluster theory to include exchange-enhancement effects in the embedding medium. Disorder in the exchange enhancement and in the local band susceptibility are treated on an equal footing. Finally, the formal connection between alloy band theory and the random-phase-approximation theory of alloy susceptibilities is elucidated. In particular, the problem of choosing the best self-consistency condition for the determination of the susceptibility $\bar{\chi}(\mathbf{q}, \omega)$ of the effective medium is considered in detail in light of the known results of alloy band theory. In the following two papers of this series, the theory presented here is extended to treat the local magnetization of alloys and the susceptibility in the presence of moments, and to include the effects of quantum and thermal spin fluctuations in a manner consistent with the results of renormalization-group theory.
\end{abstract}

\section{INTRODUCTION}

This is the first of three papers which, taken as a whole, provide a rather comprehensive theory of the saturation magnetization and the temperature- and fielddependent magnetic susceptibility of paramagnetic and ferromagnetic substitutionally disordered alloys. Although, as in all previous theories not based on the phenomenological Heisenberg model, interactions between magnetic clusters and the destruction of those clusters by internal thermal spin fluctuations are not considered, these three papers go beyond previously published theories in several important respects. First, the effects of disorder in the band susceptibility and of disorder in exchange enhancement are treated on an equal footing within a cluster theory which allows the exact embedding of a cluster in a uniquely determined best coherent-potential-approximation (CPA) effective medium. Also, a simplified method for the calculation of magnetic moments is presented, and the effect of moment formation on the susceptibility is treated quantitatively for the first time. Finally, the results of renormalization-group theory for the temperaturedependent susceptibility of a single magnetic impurity ${ }^{1}$ are extended phenomenologically to the case of magnetic clusters in a concentrated alloy.
In this first paper we present two alternative formulations of the theory of the zero-temperature, low-field susceptibility within the random-phase approximation (RPA). The first of these is a generalization of the Lederer and Mills ${ }^{2}$ treatment of local exchange enhancement for a single impurity. The second is based upon techniques developed by two of the authors ${ }^{3}$ for the cluster generalization of the coherent-potential approximation $^{4}$ and the inclusion of off-diagonal disorder (ODD) $)^{5,6}$ within the CPA. It allows the unique determination of a "best" self-consistently determined effective medium. Both formulations allow the treatment of disorder in the intra-atomic Coulomb interaction, $U$ (diagonal disorder), and disorder in the local band susceptibility, $\Gamma_{i j}$ (ODD), as well as the effects of short-range order. In the second paper we present a new theory for the dependence on local environment of the size of spontaneous moments and of the local magnetization induced by high fields, and we generalize the susceptibility formalism of this paper so as to make it valid even in the presence of spontaneous moments and/or large applied magnetic fields. In the third paper we modify the zero-temperature RPA theory of the first two papers so as to include the effect of quantum and thermal spin fluctuations in a way consistent with the results of Krishnamurthy et al. ${ }^{1}$ for the singleimpurity problem. In a fourth paper we present the re- 
sults of numerical calculations for $\mathrm{Pd}-\mathrm{Ni}$ alloys based on the theory of the first three papers and compare the results with experiment. The results found in that paper confirm that the theory presented in the first three papers yields numerical results qualitatively superior to those given by previous theories.

This series of papers was motivated by the vast amount of experimental data on alloy systems which are paramagnetic at some concentrations and ferromagnetic at others, none of which has been treated satisfactorily from a theoretical point of view. In particular, it was motivated by the failure of all previous theoretical treatments to explain, even qualitatively, the local susceptibility and magnetization of such systems, especially near the critical concentration for ferromagnetism. ${ }^{7}$ Only the phenomenological treatment of Kato and Mathon ${ }^{8}$ based on the Ginzburg-Landau theory has been able to treat even the uniform susceptibility qualitatively correctly near the critical concentration, and no previous theory has even attempted to treat the nonuniform local magnetization of such alloys. However, the theory presented in these papers is applicable to all metallic alloys, although it is perhaps of greatest value for alloy systems which are paramagnetic at some concentrations and ferromagnetic at others, especially alloys such as $\mathrm{Cu}-\mathrm{Ni}$, Pd-Ni, Pt-Ni, and Rh-Ni.

A cluster theory is required because no single-site theory can describe the effects of local fluctuations away from the uniform effective medium in which single atoms are embedded. Furthermore, because in many cases the local susceptibility, $\chi_{i j}(\omega)$, depends very strongly and nonlinearly on the local environment of the sites $i$ and $j$ (and on the region between sites $i$ and $j$ ), fluctuations in local environment can greatly affect the ensemble-averaged local susceptibilities, $\left\langle\chi_{i j}(\omega)\right\rangle$, and the uniform static susceptibility $\chi(0)$, especially near the critical concentration for any type of magnetic ordering.

We again remind the reader that the modification of the RPA results obtained in the first two of this series of papers so as to incorporate the effects of spin fluctuations, as is done in the third paper, is in most cases not merely a desirable improvement, but rather a fundamental and essential change. Thus, we caution the reader not to use the results of these two papers for the susceptibility $\chi(\omega)$ without first incorporating the effects of spin fluctuations. The reasons are as follows: As in all RPA theories, the formalisms developed in these two papers yield a divergence in the local susceptibility $\chi_{i j}(0)$ for all $i$ and $j$ whenever the local exchange enhancement reaches a critical value in the vicinity of any site, $k$. Within a single-site RPA theory this divergence gives rise to a spurious divergence in the uniform static susceptibility, $\chi(0)$, at some critical concentration $c_{\mathrm{cr}}$, to a serious overestimate of $\chi(0)$ for concentrations near $c_{\mathrm{cr}}$, and to a spurious band ferromagnetism for $c>c_{\mathrm{cr}}$. In reality, there is no critical concentration at which the static exchange-enhanced Pauli band susceptibility diverges, and ferromagnetism for $c>c_{\mathrm{cr}}$ arises from percolation among overlapping locally magnetic clusters in which fluctuations decrease with increasing overlap. For a cluster RPA theory, such as that presented here, the situation is even worse. Within such a theory there exists a different critical concentration, $c_{\mathrm{cr}}\left(C_{n}\right)$, at which $\chi(0)$ diverges for every inequivalent cluster configuration, $C_{n}$, which contains one or more possibly magnetic atoms. Thus, such a theory must yield values for $\chi(0)$ which are significantly too large over a large range of concentrations. In the limit of infinite cluster sizes, any RPA cluster theory must yield an infinite susceptibility at all concentrations for any alloy system which is magnetic over any range of concentrations. On the other hand, as is shown in the third paper of this series, the actual temperature-dependent susceptibility, $\chi_{n}(0 ; T)$, associated with any given cluster of atoms, $C_{n}$, including the effects of local thermal and quantum spin fluctuations, can be expressed solely as a function of temperature and of the RPA cluster susceptibility, $\chi_{n}^{\mathrm{RPA}}(0)$, calculated using the formalism of these two papers.

In this paper we assume that the band susceptibilities $\Gamma_{i j}^{m m^{\prime}}(\omega)$ which are the partial derivatives of the magnetization of the orbitals $m$ on the sites $i$ with respect to the magnetic fields applied to the orbitals $m^{\prime}$ on the sites $j$ in the absence of moments, are known. They can be calculated from the equation

$$
\begin{aligned}
\Gamma_{i j}^{m m^{\prime}}(\omega) & \equiv\left(\partial \mu_{i m} / \partial H_{j m^{\prime}}\right)_{\left\{\mu_{l m^{\prime \prime}}=0 \text { for } l, m^{\prime \prime} \neq i, m\right\}} \\
& =(1 / \pi) \operatorname{Im} \int_{-\infty}^{\varepsilon_{F}} d \varepsilon G_{i j}^{m m^{\prime}}(\varepsilon) G_{i j}^{m m^{\prime}}(\varepsilon+\omega),
\end{aligned}
$$

provided that the Green's functions $G_{i j}^{m m^{\prime}}$ are known from an alloy band calculation. Within a site representation one obtains the integral equation

$$
\chi_{i j}^{m m^{\prime}}(\omega)=\Gamma_{i j}^{m m^{\prime}}(\omega)+\sum_{k, m^{\prime \prime}, m^{\prime \prime \prime}} \Gamma_{i k}^{m m^{\prime \prime}}(\omega) u_{k}^{m m^{\prime \prime} m^{\prime \prime \prime}} \chi_{k j}^{m^{\prime \prime \prime} m^{\prime}}(\omega),
$$

which is derived in Appendix A. For a $(2 l+1)$-fold band one finds the result

$$
\sum_{m^{\prime}} u_{i}^{m m^{\prime}}=U_{i}+2 l J_{i} \equiv(2 l+1) u_{i}
$$

in the simple limit in which $u_{i}^{m m^{\prime}} \equiv u_{i}$ and in which the $J_{i}^{m m^{\prime}} \equiv J_{i}$ are independent of $m$ and $m^{\prime}$. In this limit, if it is also true that the matrix elements $\Gamma_{i j}^{m m^{\prime}}(\omega)$ are of the form

$\Gamma_{i j}^{m m^{\prime}}(\omega)=\delta_{m m^{\prime}}, \Gamma_{i j}^{\mathrm{diag}}(\omega)+\left(1-\delta_{m m^{\prime}}\right) \Gamma_{i j}^{\mathrm{off}-\operatorname{diag}}(\omega)$,

one finds the result

$$
\begin{aligned}
\chi_{i j}(\omega) & \equiv \sum_{m, m^{\prime}} \chi_{i j}^{m m^{\prime}}(\omega) \\
& =\Gamma_{i j}(\omega)+\sum_{k} \Gamma_{i k}(\omega) u_{k} \chi_{k j}(\omega),
\end{aligned}
$$

where $\Gamma_{i j}(\omega) \equiv \sum_{m, m^{\prime}} \Gamma_{i j}^{m m^{\prime}}(\omega)$. In this paper we assume the approximate validity of Eqs. (1.2) and (1.4) (or, equivalently, consider the case of a single nondegenerate band) and take the well-known Eq. (1.5) as our basic starting point. The $s$ and $p$ bands are neglected because their exchange enhancement is known to be very small. The formalism given in this paper is applicable in a 
straightforward manner to the solution of the exact Eq. (1.2) for the $\chi_{i j}^{m m^{\prime}}(\omega)$ with any number of bands and orbitals. However, in that general case the numerical determination of $\chi_{i j}^{m m^{\prime}}(\omega)$ requires substantially more computational labor.

In Sec. II of this paper we generalize the localexchange-enhancement theory of Lederer and Mills ${ }^{2}$ so as to make it applicable to concentrated alloys and so as to treat ODD on an equal footing with diagonal disorder, either within the geometric approximation or in the general case. The treatment given in that section is applicable to the treatment either of a number of impurities embedded in a pure host metal or of a compact cluster of real atoms embedded in a given effective medium. However, that treatment does not suffice for the best self-consistent determination of an effective medium. In Sec. III alloy band-theory techniques developed by two of the authors ${ }^{3}$ are used to develop alternative formalisms for the susceptibility. Both renormalized-interactor and $t$-matrix formalisms are presented for the case of diagonal disorder and for that of ODD. The selfconsistent determination of a cluster-CPA effective medium is considered in Sec. IV. Finally, in Sec. V our results are summarized and discussed critically.

\section{GENERALIZATION OF LOCAL EXCHANGE ENHANCEMENT}

In this section we present methods for solving the susceptibility integral equation (1.5) for three different classes of concentrated alloys. First, we consider the case of diagonal disorder - the case of alloys for which the unenhanced band susceptibilities, $\Gamma_{i j}(\omega)$, are identical for the constituent metals so that $\Gamma_{i j}(\omega)$ for the alloy is translationally invariant, but in which the intra-atomic Coulomb interaction, $u_{k}$, differs for the different alloy constituents (diagonal disorder). Second, we consider the case in which the $\Gamma_{i j}$ 's, as well as the $u_{k}$ 's, can be different for different constituents (diagonal and offdiagonal disorder), but in which the band susceptibilities must satisfy the relation

$$
\left[\Gamma_{i j}^{A B}(\omega)\right]^{2}=\Gamma_{i j}^{A A}(\omega) \Gamma_{i j}^{B B}(\omega),
$$

where $A$ and $B$ denote any two alloy constituents (the geometric approximation). Finally, we consider the case of alloys characterized by both diagonal and general offdiagonal disorder, with the $\Gamma_{i j}$ 's satisfying no particular relation.

We consider the case of $n$ impurities embedded in a pure host, or its formal equivalent, the case of $n$ real atoms embedded in an exchanged-enhanced effective medium. The formalism presented is a straightforward generalization of the single-impurity local-exchangeenhancement theory of Lederer and Mills. ${ }^{2}$ However, it is the first cluster theory of alloy susceptibilities to treat exactly the embedding of a cluster in either a pure host or an effective medium. Also, it is the first such theory to take account of exchange enhancement in the host or embedding medium.

\section{A. Diagonal disorder}

Following Lederer and Mills, in this subsection we assume the band susceptibility to be translationally invariant,

$$
\Gamma_{i j}(\omega)=\Omega^{-1} \int_{\mathrm{BZ}} \Gamma(\overrightarrow{\mathbf{q}}, \omega) e^{-i \overrightarrow{\mathbf{q}} \cdot \overrightarrow{\mathbf{R}}_{i j}} d^{3} q,
$$

where $\Omega$ is the volume of the Brillouin zone $(B Z)$ of the lattice, $\overrightarrow{\mathbf{R}}_{i j}=\overrightarrow{\mathbf{R}}_{i}-\overrightarrow{\mathbf{R}}_{j}$ is the position vector from site $j$ to site $i$, and the integral is over the Brillouin zone.

It is useful to define the quantity $\delta u_{k}(\omega) \equiv u_{k}-\bar{u}(\omega)$, where $\bar{u}(\omega)$ is the value of the intra-atomic Coulomb interaction in the host or effective medium. For the case of $n$ impurities embedded in a pure host, $u(\omega)$ is just the value of $u_{k}$ in the host metal and is assumed to be frequency independent, whereas for the case of a cluster of $n$ real atoms embedded in an effective medium, $\bar{u}(\omega)$ is the frequency-dependent self-consistently determined value of $u_{k}$ for the medium. In terms of $\delta u_{k}(\omega)$, the integral equation for the susceptibility, Eq. (1.5), can be written in the form

$$
\begin{aligned}
\chi_{i j}(\omega)= & \Gamma_{i j}(\omega)+\bar{u}(\omega) \sum_{k} \Gamma_{i k}(\omega) \chi_{k j}(\omega) \\
& +\sum_{k} \Gamma_{i k}(\omega) \delta u_{k}(\omega) \chi_{k j}(\omega)
\end{aligned}
$$

Upon taking the Fourier transform (FT) of this equation, one obtains the expression

$$
\chi\left(\overrightarrow{\mathbf{q}}, \overrightarrow{\mathbf{q}}^{\prime} ; \omega\right)=\bar{\chi}(\overrightarrow{\mathbf{q}} ; \omega)\left[\delta_{\overrightarrow{\mathbf{q}} \overrightarrow{\mathbf{q}}^{\prime}}+N^{-1} \sum_{k, l} \chi_{k l}(\omega) \delta u_{k}(\omega) \exp \left[i\left(\overrightarrow{\mathbf{q}} \cdot \overrightarrow{\mathbf{R}}_{k}-\overrightarrow{\mathbf{q}}^{\prime} \cdot \boldsymbol{R}_{l}\right)\right]\right]
$$

where $N$ is the total number of sites in the lattice and where

$$
\bar{\chi}(\overrightarrow{\mathbf{q}} ; \omega)=\Gamma(\overrightarrow{\mathbf{q}} ; \omega) /[1-\bar{u}(\omega) \Gamma(\overrightarrow{\mathbf{q}} ; \omega)]
$$

is the enhanced susceptibility of the host (or the effective medium). The inverse FT of Eq. (2.4) is then given by the expression

$$
\chi_{i j}(\omega)=\bar{\chi}_{i j}(\omega)+\sum_{k} \bar{\chi}_{i k}(\omega) \delta u_{k}(\omega) \chi_{k j}(\omega)
$$

In order to invert this equation and solve for $\chi_{i j}(\omega)$, we note that the sum over $k$ extends only over the $n$ impurity sites (or $n$ real-atom sites in an effective medium). Upon confining $i$ to the subspace $C$ of impurity sites, Eq. (2.6) assumes the form

$$
\vec{\chi}_{j}(\omega)=\overrightarrow{\bar{\chi}}_{j}(\omega)+\bar{\chi}(\omega) \delta \mathbf{u}(\omega) \vec{\chi}_{j}(\omega)
$$

where the vectors $\vec{\chi}_{j}(\omega)$ and $\overrightarrow{\bar{\chi}}_{j}(\omega)$ have components $\chi_{k j}(\omega)$ and $\bar{\chi}_{k j}(\omega)$, respectively, and the matrices $\bar{\chi}(\omega)$ 
and $\delta \mathbf{u}(\omega)$ have elements $\bar{\chi}_{k l}(\omega)$ and $\delta u_{l m} \equiv \delta_{l m} \delta u_{l}$, with the sites $k, l$, and $m$ confined to the subspace $C$. Equation (2.7) is easily inverted, yielding the solution

$$
\vec{\chi}_{j}(\omega)=\mathbf{A}^{-1}(\omega) \overrightarrow{\bar{\chi}}_{j}(\omega)
$$

or, equivalently,

$$
\chi_{k j}(\omega)=\sum_{l}\left[\mathbf{A}^{-1}(\omega)\right]_{k l} \bar{\chi}_{l j}(\omega),
$$

where the matrix to be inverted,

$$
\mathbf{A}(\omega)=1-\bar{\chi}(\omega) \boldsymbol{\delta} \mathbf{u}(\omega),
$$

is defined only on the subspace $C$, so that the summation over $l$ in Eq. (2.9) extends only over that subspace. Inserting Eq. (2.9) into Eq. (2.6), one finds the expression

$$
\chi(\omega)=\bar{\chi}(\omega)\left[1+\delta \mathbf{u}(\omega) \mathbf{A}^{-1}(\omega) \bar{\chi}(\omega)\right]
$$

for the exchange-enhanced local susceptibility. The uniform susceptibility is then given by the expression

$$
\begin{aligned}
\chi(\omega) & \equiv N^{-1} \sum_{i, j} \chi_{i j}(\omega) \\
& =\bar{\chi}(\omega)\left[1+N^{-1} \bar{\chi}(\omega) \sum_{k, l} \delta u_{k}(\omega)\left[\mathbf{A}^{-1}(\omega)\right]_{k l}\right),
\end{aligned}
$$

where $\bar{\chi}(\omega)$ is the uniform susceptibility of the host (or the effective medium). Finally, the fractional change in the uniform susceptibility introduced by the $n$ impurities (or the $n$ real atoms in an effective medium) is given by

$\delta \chi(\omega) / \bar{\chi}(\omega)=N^{-1} \bar{\chi}(\omega) \sum_{k, l} \delta u_{k}(\omega)\left[\mathbf{A}^{-1}(\omega)\right]_{k l}$.

Equation (2.13) provides an easy method for the calculation of $\delta \chi(\omega)$ for any configuration of $n$ impurities dissolved in an exchange-enhanced host (or effective medium). The numerical calculation of $\delta \chi(\omega)$ involves only the inversion of a single $n \times n$ matrix, provided that the local susceptibilities, $\bar{\chi}_{i j}(\omega)$, of the host (or the effective medium) are known. For the case of an isolated impurity on site 0 and for $\omega=0$, Eqs. (2.10)-(2.13) reduce to the results of Lederer and Mills.

\section{B. Diagonal and off-diagonal disorder within the geometric approximation}

In this subsection, the formalism of Sec. II A is generalized so as to be made applicable to the case of disordered alloys characterized by diagonal and off-diagonal disorder within the geometric approximation. In general, the off-diagonal band susceptibilities, $\Gamma_{i j}(\omega)$, depend not only on the chemical occupation of the sites, $i$ and $j$, but also on the local environment of those sites. In principle, it is possible to treat this general depen- dence $\Gamma_{i j}(\omega)$ on the local environment. However, although the formalism for such a treatment is conceptually straightforward, its computational implementation is quite difficult. Thus, in this subsection, as in all previous treatments of ODD, $\Gamma_{i j}(\omega)$ is assumed to be a function only of the occupation of the sites $i$ and $j$; i.e., the matrix elements $\Gamma_{i j}(\omega)$ are assumed to take on only values of the type $\Gamma_{i j}^{A A}(\omega), \Gamma_{i j}^{A B}(\omega)=\Gamma_{i j}^{B A}(\omega)$, and $\Gamma_{i j}^{B B}(\omega)$. A further great computational savings can be made by assuming that the local band susceptibilities, $\Gamma_{i j}(\omega)$, depend on the atomic occupation of sites $i$ and $j$ only within the geometric approximation of Shiba, ${ }^{9}$ Eq. (2.1). Within this approximation one can write

$$
\Gamma_{i j}(\omega)=\eta_{i}(\omega) \bar{\Gamma}_{i j}(\omega) \eta_{j}(\omega),
$$

where

$$
\eta_{i}(\omega)=\left[\Gamma_{i i}(\omega) / \bar{\Gamma}_{i i}(\omega)\right]^{0.5},
$$

and where $\bar{\Gamma}_{i j}(\omega)$ is the local band susceptibility of the host or embedding medium. This off-diagonal disorder is dominant for such isovalent alloys as $\mathrm{Pd}-\mathrm{Ni}$ and $\mathrm{Pt}-\mathrm{Ni}$, and the geometric approximation renders its treatment feasible without the use of a supercomputer.

Inserting Eq. (2.14) into Eq. (1.5), one obtains the result

$$
\begin{aligned}
\chi_{i j}(\omega)= & \eta_{i}(\omega) \bar{\Gamma}_{i j}(\omega) \eta_{j}(\omega) \\
& +\sum_{k} \eta_{i}(\omega) \bar{\Gamma}_{i k}(\omega) \eta_{k}(\omega) u_{k} \chi_{k j}(\omega) .
\end{aligned}
$$

Upon defining the quantity

$$
\xi_{i j}(\omega) \equiv\left[\eta_{i}(\omega)\right]^{-1} \chi_{i j}(\omega)\left[\eta_{j}(\omega)\right]^{-1},
$$

dividing Eq. (2.16) by $\eta_{i}(\omega) \eta_{j}(\omega)$, and substituting Eq. (2.17) into the resultant equation, one finds

$$
\xi_{i j}(\omega)=\bar{\Gamma}_{i j}(\omega)+\sum_{k} \bar{\Gamma}_{i k}(\omega)\left[\eta_{k}(\omega)\right]^{2} u_{k} \xi_{k j}(\omega) .
$$

Then, upon defining the quantity

$$
\delta u_{k}^{\mathrm{eff}}(\omega) \equiv\left[\eta_{k}(\omega)\right]^{2} u_{k}-\bar{u}(\omega),
$$

which is analogous to the quantity $\delta u_{k}(\omega)$, defined in Sec. II A, one finds that Eq. (2.18) assumes the form

$$
\begin{aligned}
\xi_{i j}(\omega)= & \bar{\Gamma}_{i j}(\omega)+\bar{u}(\omega) \sum_{k} \bar{\Gamma}_{i k}(\omega) \xi_{k j}(\omega) \\
& +\sum_{k} \bar{\Gamma}_{i k}(\omega) \delta u_{k}^{\mathrm{eff}}(\omega) \xi_{k j}(\omega)
\end{aligned}
$$

This equation for $\xi_{i j}(\omega)$ is exactly analogous to Eq. (2.3) of Sec. II A for $\chi_{i j}(\omega)$, with $\delta u_{k}(\omega)$ being replaced by $\delta u_{k}^{\text {eff }}(\omega)$, and thus can be solved in exactly the same way that Eq. (2.3) was solved. One finds the result

$$
\chi_{i j}(\omega)=\eta_{i}(\omega) \eta_{j}(\omega)\left\{\bar{\chi}_{i j}(\omega)+\sum_{k, l} \bar{\chi}_{i k}(\omega) \delta u_{k}^{\mathrm{eff}}(\omega)\left\{\left[\mathbf{A}^{\mathrm{eff}}(\omega)\right]^{-1}\right\}_{k l} \bar{\chi}_{l j}(\omega)\right]
$$


where $\mathbf{A}^{\text {eff }}(\omega)$ is defined in exactly the same way as the matrix $\mathbf{A}(\omega)$ was defined in Eq. (2.10) of Sec. II A except for the replacement of $\delta \mathbf{u}(\omega)$ by $\delta \mathbf{u}^{\text {eff }}(\omega)$, and where the summations over $k$ and $l$ again extend only over the subspace $C$. Thus, the fractional change in the uniform susceptibility is given by the expression

$$
\begin{aligned}
& \delta \chi(\omega) / \bar{\chi}(\omega)=N^{-1} \sum_{k, l}[ \bar{\chi}(\omega) \delta u_{k}^{\mathrm{eff}}(\omega)\left\{\left[\mathbf{A}^{\mathrm{eff}}(\omega)\right]^{-1}\right\}_{k l} \\
&+\left[\eta_{k}(\omega)-1\right] \sum_{m}\left(\left\{1+\bar{\chi}(\omega) \delta \mathbf{u}^{\mathrm{eff}}(\omega)\left[\mathbf{A}^{\mathrm{eff}}(\omega)\right]^{-1}\right\}_{k m}\right. \\
&\left.\left.\quad \times\left\{2 \delta_{m l}+\left[\eta_{l}(\omega)-1\right] \bar{\chi}_{m l}(\omega) / \bar{\chi}(\omega)\right\}\right)\right\} .
\end{aligned}
$$

Note that in the absence of off-diagonal disorder Eq. (2.22) reduces to the corresponding Eq. (2.13) of Sec. II A. Furthermore, although the form of this expression is slightly more complicated than that of Eq. (2.13), it provides an equally easy method for the numerical calculation of $\delta \chi(\omega) / \bar{\chi}(\omega)$, requiring only the inversion of a single $n \times n$ matrix.

\section{Diagonal and general off-diagonal disorder}

In this subsection the formalism of Sec. II A is generalized so as to be made applicable to the case of disordered alloys characterized by any type of disorder, including general off-diagonal disorder. In contrast to the preceding subsection, no particular relationship is assumed to exist between the different possible values of $\Gamma_{i j}(\omega)$, and those values are allowed, in principle, to de- pend on the local environment of sites $i$ and $j$, as well as on the chemical occupation of sites $i$ and $j$.

As in Sec. II A and II B, we consider the case of $n$ impurity atoms embedded in a pure host, or of $n$ real atoms embedded in an effective medium. We also consider that an additional $n^{\prime}$ atoms of the embedding medium are connected to the impurity atoms by matrix elements $\Gamma_{k l}$ different from the matrix elements $\bar{\Gamma}_{k l}$ of the translationally invariant band susceptibility of the embedding medium. In general, the number $n^{\prime}$ of such atoms will be large even for small $n$. We then assume all matrix elements $\Gamma_{i j}$ to be equal to $\bar{\Gamma}_{i j}$ if either site $i$ or site $j$ lies outside the subspace $C^{\prime}$ consisting of the $n$ sites occupied by impurity atoms and the additional $n^{\prime}$ sites defined above, thus neglecting the dependence of $\Gamma_{i j}$ on local environment if either site lies outside $C^{\prime}$.

In this case Eq. (1.5) can be written in the form

$$
\chi_{i j}(\omega)=\bar{\Gamma}_{i j}(\omega)+\delta \Gamma_{i j}(\omega)+\bar{u}(\omega) \sum_{k} \bar{\Gamma}_{i k}(\omega) \chi_{k j}(\omega)+\sum_{k}\left[\bar{u}(\omega) \delta \Gamma_{i k}(\omega)+\bar{\Gamma}_{i k}(\omega) \delta u_{k}(\omega)\right] \chi_{k j}(\omega)
$$

The FT of this equation is given by

$$
\begin{aligned}
\chi\left(\overrightarrow{\mathbf{q}}, \overrightarrow{\mathbf{q}}^{\prime} ; \omega\right)= & \bar{\chi}(\overrightarrow{\mathbf{q}} ; \omega) \delta_{\overrightarrow{\mathbf{q}} \overrightarrow{\mathbf{q}}^{\prime}}+N^{-1} \bar{\alpha}(\overrightarrow{\mathbf{q}} ; \omega) \sum_{l, m} \delta \Gamma_{l m}(\omega) \exp \left[i\left(\overrightarrow{\mathbf{q}} \cdot \overrightarrow{\mathbf{R}}_{l}-\mathbf{q}^{\prime} \cdot \overrightarrow{\mathbf{R}}_{m}\right)\right] \\
& +N^{-1} \bar{\alpha}(\overrightarrow{\mathbf{q}} ; \omega) \sum_{k, l, m}\left[\bar{u}(\omega) \delta \Gamma_{l k}(\omega)+\bar{\Gamma}_{l k}(\omega) \delta u_{k}(\omega)\right] \chi_{k m}(\omega) \exp \left[i\left(\overrightarrow{\mathbf{q}} \cdot \overrightarrow{\mathbf{R}}_{l}-\mathbf{q}^{\prime} \cdot \overrightarrow{\mathbf{R}}_{m}\right)\right],
\end{aligned}
$$

where $k, l$, and $m$ are confined to the subspace $C^{\prime}$, $\bar{\chi}(\overrightarrow{\mathbf{q}} ; \omega)$ is the exchange-enhanced susceptibility of the host (or effective medium) given by Eq. (2.5), and

$$
\begin{aligned}
\bar{\alpha}(\overrightarrow{\mathbf{q}} ; \omega) & \equiv[1-\bar{u}(\omega) \bar{\Gamma}(\overrightarrow{\mathbf{q}} ; \omega)]^{-1} \\
& =\bar{\chi}(\overrightarrow{\mathbf{q}} ; \omega) / \bar{\Gamma}(\overrightarrow{\mathbf{q}} ; \omega) .
\end{aligned}
$$

The inverse FT of Eq. (2.24) is then given by

$$
\begin{aligned}
\chi_{i j}(\omega)=\bar{\chi}_{i j}(\omega)+\sum_{k} \bar{\alpha}_{i k} \delta \Gamma_{k j}(\omega) & \\
& +\sum_{k, l} \bar{\alpha}_{i k}(\omega)\left[\bar{u}(\omega) \delta \Gamma_{k l}(\omega)\right. \\
& \left.+\bar{\Gamma}_{k l}(\omega) \delta u_{l}(\omega)\right] \chi_{l j}(\omega) .
\end{aligned}
$$

In solving Eq. (2.26) it is necessary to invert a matrix $\mathbf{A}(\omega)$, defined on the supercluster subspace $C^{\prime}$ of $n+n^{\prime}$ sites. Following the same procedure as that used in solving Eq. (2.6) in Sec. II A, one finds the result

$\chi_{k j}(\omega)=\sum_{l}\left[\mathbf{A}^{-1}(\omega)\right]_{k l}\left[\bar{\chi}_{l j}(\omega)+\sum_{m} \bar{\alpha}_{l m}(\omega) \delta \Gamma_{m j}(\omega)\right]$,

where $k, l$, and $m$ are restricted to the subspace $C^{\prime}$ and the matrix $\mathbf{A}$ is defined by the equation

$$
\mathbf{A}(\omega) \equiv \mathbf{1}-\overline{\boldsymbol{\alpha}}(\omega)[\overline{\mathbf{u}}(\omega) \boldsymbol{\delta} \boldsymbol{\Gamma}(\omega)+\Gamma(\omega) \boldsymbol{\delta} \mathbf{u}(\omega)] .
$$

Finally, substituting Eq. (2.27) into Eq. (2.26), one obtains the expressions 


$$
\chi(\omega)=\bar{\chi}(\omega)+\overline{\boldsymbol{\alpha}}(\omega)\left\{\delta \boldsymbol{\Gamma}(\omega)+[\overline{\mathbf{u}}(\omega) \delta \boldsymbol{\Gamma}(\omega)+\boldsymbol{\Gamma}(\omega) \boldsymbol{\delta} \mathbf{u}(\omega)] \mathbf{A}^{-1}(\omega)[\bar{\chi}(\omega)+\overline{\boldsymbol{\alpha}}(\omega) \boldsymbol{\delta} \boldsymbol{\Gamma}(\omega)]\right\}
$$

for the exchange-enhanced local susceptibility and

$$
\delta \chi(\omega) / \bar{\chi}(\omega)=\delta \Gamma(\omega) / \bar{\Gamma}(\omega)+N^{-1} \bar{\alpha}(\omega) \sum_{k, l}\left\{[\overline{\mathbf{u}}(\omega) \boldsymbol{\delta} \boldsymbol{\Gamma}(\omega)+\overline{\boldsymbol{\Gamma}}(\omega) \boldsymbol{\delta} \mathbf{u}(\omega)] \mathbf{A}^{-1}(\omega)[\mathbf{1}+\overline{\boldsymbol{\Gamma}}-1(\omega) \boldsymbol{\delta} \boldsymbol{\Gamma}(\omega)]\right\}_{k l}
$$

for the fractional change in the uniform susceptibility of the alloy, where $\bar{\Gamma}(\omega)$ is the uniform band susceptibility of the host or effective medium.

It is clear that the computational application of the formalism of this subsection is substantially more time consuming than is that of Secs. II A and II B, because of the large dimensionality of the matrix $\mathbf{A}(\omega)$ to be inverted. In order to apply this formalism without the use of a supercomputer one must truncate the matrix elements $\delta \Gamma_{i j}$ at distances of the order of third-nearest-neighbor distances. The use of a supercomputer would allow one to extend the $\delta \Gamma_{i j}$ to somewhat larger distances. In the following section we present an alternative, more sophisticated, but computationally more feasible formalism for the calculation of $\bar{\chi}(\omega)$ and $\delta \chi(\omega) / \bar{\chi}(\omega)$. That formalism, which draws upon the techniques of CPA theories ${ }^{5,6}$ for the band structure of alloys characterized by off-diagonal disorder, involves the inversion of a $2 n \times 2 n$ matrix rather than an $\left(n+n^{\prime}\right) \times\left(n+n^{\prime}\right)$ matrix.

\section{APPLICATION OF ALLOY-BAND-THEORY TECHNIQUES TO THE DETERMINATION OF $\chi_{i j}(\omega)$}

In this section we present an alternative formalism for the solution of the susceptibility integral equation (1.5) analogous to the cluster-CPA formalism of Ref. 3. In Sec. III A we briefly compare the alloy-band-theory equation of motion for Green's function, $G_{i j}(\omega)$, with the integral equation for $\chi_{i j}(\omega)$ and discuss the differences and similarities between those two equations. We show that although the susceptibility equation is of a different form, it can be transformed into a form exactly analogous mathematically to that of the Green'sfunction equation. In Secs. III B and III C we develop renormalized-interactor and $t$-matrix formalisms which allow one to determine the exchange-enhanced susceptibility of a cluster embedded in an effective medium for the case of diagonal disorder. For this case the results obtained using these techniques are identical to those found in Sec. II. Finally, in Sec. III D these two formalisms are generalized so as to allow the $\Gamma_{i j}(\omega)$ to depend on the chemical occupation of the sites $i$ and $j$ (ODD). In the presence of ODD, these techniques do yield new and more useful results than those obtained in Sec. II.

\section{A. Comparison of the equations for $\chi_{i j}(\omega)$ and for $G_{i j}(\omega)$}

In a site (or cluster) representation the single-particle Green's function, $\boldsymbol{G}_{i j}(\omega)$, satisfies the equation of motion

$$
G_{i j}(\omega)=g_{i}(\omega)\left[\delta_{i j}+\sum_{k} W_{i k} G_{k j}(\omega)\right],
$$

or, in matrix form,

$$
\mathbf{G}(\omega)=\mathbf{g}(\omega)[1+\mathbf{W G}(\omega)] .
$$

Here,

$$
g_{i}(\omega) \equiv\left(\omega-\varepsilon_{i}\right)^{-1}
$$

is the "bare" Green's function (or locator) for the site (or cluster) $i$. In a site representation all of the quantities in Eq. (3.1a) are scalars; in a cluster representation with $n$ site clusters, they all are $n \times n$ matrices with elements $G_{i j, \alpha \beta} \equiv\left(\mathbf{G}_{i j}\right)_{\alpha \beta}, \quad g_{i, \alpha \beta} \equiv\left(\mathbf{g}_{i}\right)_{\alpha \beta}, \quad$ and $\quad W_{i k, \alpha \beta} \equiv\left(\mathbf{W}_{i k}\right)_{\alpha \beta}$, where $\alpha$ and $\beta$ run from 1 to $n$. Upon rewriting Eq. (1.5) in matrix notation,

$$
\chi(\omega)=\Gamma(\omega)[1+\mathbf{u} \chi(\omega)],
$$

one finds that this equation is of the same form as Eq. (3.1b) for $\mathbf{G}(\omega)$. However, it differs in that $\Gamma(\omega)$ contains nonzero off-diagonal elements, whereas $g(\omega)$ is diagonal, and in that $\mathbf{u}$ is purely diagonal, whereas $\mathbf{W}$ is purely off diagonal. Thus, solutions of Eq. (1.5) cannot be obtained directly by analogy with the alloy-bandtheory solutions of Eq. (3.1). However, as we now show, Eq. (1.5) can be transformed to a form directly analogous to Eq. (3.1).

One can reexpress Eq. (3.3) in the form

$$
\boldsymbol{S}(\omega)=\boldsymbol{g}(\omega)[1+\mathcal{W}(\omega) \boldsymbol{S}(\omega)],
$$

which is identical in form to Eq. (3.1b) of alloy band theory, by multiplying Eq. (3.3) by u, adding a unit matrix on both sides, and introducing the matrices

$$
\begin{aligned}
& \boldsymbol{S}(\omega) \equiv 1+\mathbf{u} \chi(\omega), \\
& \boldsymbol{g}(\omega) \equiv[1-\mathbf{u} \gamma(\omega)]^{-1},
\end{aligned}
$$

and

$$
\mathcal{W}(\omega) \equiv \mathbf{u}[\boldsymbol{\Gamma}(\omega)-\boldsymbol{\gamma}(\omega)]
$$

where $\gamma(\omega)$ is the diagonal part of $\Gamma(\omega)$,

$$
\gamma_{i j}(\omega)=\Gamma_{i j}(\omega) \delta_{i j} \equiv \gamma_{i}(\omega)
$$

The matrix $g(\omega)$ is purely site diagonal (or cluster diagonal), $\mathscr{F}_{i j}(\omega)=\delta_{i j} \mathscr{g}_{i}(\omega)$, and has the same relation to $\mathcal{S}(\omega)$ as $\mathbf{g}(\omega)$ has to $\mathbf{G}(\omega)$ in alloy band theory, and the matrix $\mathcal{W}(\omega)$ is purely off diagonal, as is $\mathbf{W}$. Thus, one can determine $\boldsymbol{S}(\omega)$ for a cluster embedded in an effective medium by using standard techniques ${ }^{3}$ of alloy band theory and then find $\chi(\omega)$ from Eq. (3.5). However, this procedure does not necessarily yield the best selfconsistency condition for the determination of $\bar{\chi}(\omega)$ for the effective medium; procedures for determining $\bar{\chi}(\omega)$ are discussed in detail in Sec. IV. 


\section{B. Renormalized-interactor formalism for $\chi_{i j}(\omega)$ for the case of diagonal disorder}

In this subsection we modify the alloy-band-theory renormalized-interactor formalism of Gonis and Garland $^{3}$ so as to use it to derive an equation for $\chi_{i j}(\omega)$ for sites $i$ and $j$ in the cluster for the case of diagonal disorder. In the interactor formalism one solves Eq. (3.4) for

$$
g_{i i}(\omega)=\mathscr{g}_{i}(\omega)\left(1+\sum_{k} \mathcal{W}_{i k}(\omega) g_{k i}(\omega)\right)
$$

$$
\Delta_{i}(\omega) \equiv u_{i}^{-1}\left(\sum_{k} \mathcal{W}_{i k}(\omega) \mathscr{g}_{k}(\omega) \mathcal{W}_{k i}(\omega)+\sum_{k} \sum_{(\neq i)} \sum_{l \neq i, k)} \mathcal{W}_{i k}(\omega) \mathscr{g}_{k}(\omega) \mathcal{W}_{k l}(\omega) \mathscr{g}_{l}(\omega) \mathcal{W}_{l i}(\omega)+\cdots\right)
$$

is independent of the chemical occupation of the site (or cluster) $i$. The factor $u_{i}^{-1}$, which appears on the righthand side of Eq. (3.10), and which has no analogy in alloy band theory, is introduced here in order to cancel the factor $u_{i}$ in Eq. (3.7) for $\mathcal{W}_{i k}(\omega)$. Note that the inclusion of this factor in the definition of $\Delta_{i}(\omega)$ renders $\Delta_{i}$ independent of the chemical occupation of the site (or cluster) $i$.

In order to determine $S_{i i}(\omega)$ from Eq. (3.9b) and, hence, obtain $\chi_{i i}(\omega)$, one must somehow determine $\Delta_{i}(\omega)$. In order to do that, we follow the usual procedure of embedding the site (or cluster) 0 in an effective medium. Then, because $\Delta_{0}(\omega)$ is independent of the occupation of the site (or cluster) $0, \Delta_{0}(\omega)$ is equal to $\bar{\Delta}(\omega)$, the renormalized interactor for the pure, translationally invariant effective medium. Placing effective medium on site (or cluster) $i$, as well as on all other sites, one finds from Eq. (3.9b) the result

$$
\bar{\Delta}(\omega)=\bar{u}^{-1}(\omega)\left[\bar{g}^{-1}(\omega)-\overline{\mathscr{S}}_{00}^{-1}(\omega)\right],
$$

where $\bar{u}(\omega)$ denotes the value of $u_{i}(\omega)$ for the pure effective medium and $\bar{g}(\omega)$ and $\bar{S}_{00}(\omega)$ are given by the equations

$$
\overline{\mathscr{g}}(\omega)=[\underline{1}-\bar{u}(\omega) \gamma(\omega)]^{-1}
$$

and

$$
\overline{\mathscr{S}}_{00}(\omega)=1+\bar{u}(\omega) \bar{\chi}_{00}(\omega),
$$

with $\bar{\chi}_{\mathrm{O} 0}(\omega)$ obtained as the FT of the quantity

$$
\bar{\chi}(\mathbf{q}, \omega)=\Gamma(\mathbf{q}, \omega)[1-\bar{u}(\omega) \Gamma(\overrightarrow{\mathbf{q}}, \omega)]^{-1} .
$$

Upon substituting Eq. (3.11) into Eq. (3.9b), one obtains the result

$g_{00}(\omega)=\left\{\mathscr{g}_{0}^{-1}(\omega)-u_{0} \bar{u}^{-1}(\omega)\left[\overline{\mathscr{g}}^{-1}(\omega)-\overline{\mathcal{S}}_{00}^{-1}(\omega)\right]\right\}^{-1}$.

Then, upon inserting Eqs. (3.12) and (3.13) into Eq. (3.15), one finds the equation

$$
S_{00}(\omega)=\left[1-u_{0} \bar{u}^{-1}(\omega)+u_{0} \bar{u}^{-1}(\omega) \overline{\mathcal{S}}_{00}^{-1}(\omega)\right]^{-1},
$$

by performing a perturbation expansion of the last term on the right-hand side of Eq. (3.8) in powers of $\mathcal{W}(\omega)$. As is shown in Ref. 3, one finds the result

$$
\mathcal{S}_{i i}(\omega)=\mathscr{g}_{i}\left[1+u_{i} \Delta_{i}(\omega) \mathcal{S}_{i i}(\omega)\right],
$$

or, equivalently,

$$
g_{i i}(\omega)=\left[\mathscr{g}_{i}^{-1}(\omega)-u_{i} \Delta_{i}(\omega)\right]^{-1} .
$$

Here, the fully renormalized interactor,

or, equivalently, in terms of the site (or cluster) quantity, $\delta u_{0}(\omega) \equiv u_{0}-\bar{u}(\omega)$,

$$
\mathcal{S}_{00}(\omega)=\bar{S}_{00}(\omega)\left[1-\delta u_{0}(\omega) \bar{\chi}_{00}(\omega)\right]^{-1} .
$$

Finally, using Eq. (3.12) one obtains the desired equation,

$$
\begin{aligned}
\chi_{00}(\omega) & =\bar{\chi}_{00}(\omega)\left[1-\delta u_{0}(\omega) \bar{\chi}_{00}(\omega)\right]^{-1} \\
& =\left[1-\bar{\chi}_{00}(\omega) \delta u_{0}(\omega)\right]^{-1} \bar{\chi}_{00}(\omega) .
\end{aligned}
$$

Within the cluster subspace, this equation for the susceptibility is equivalent to Eq. (2.9), found in site representation in Sec. II purely by FT techniques. Although this technique does not yield a formula for $\chi_{i j}(\omega)$ valid outside the cluster subspace, the $t$-matrix theory of the next subsection does yield such a formula valid for all $i$ and $j$.

\section{T-matrix formalism for $\chi_{i j}(\omega)$ \\ for the case of diagonal disorder}

In this subsection we derive the $t$ matrix for the exchange-enhanced $d$ band local susceptibility, $\chi_{i j}(\omega)$, for the case of diagonal disorder for a single site (or cluster) embedded in an effective medium. We use that $t$ matrix to rederive Eq. (2.11), which, unlike Eq. (3.17), is valid for all of the elements of $\chi(\omega)$, not only the cluster-diagonal elements. The $t$-matrix formalism for $\chi_{i j}(\omega)$, which we introduce here, is exactly analogous to the usual $t$-matrix formalism for the Green's function, $G_{i j}(\omega)$, of alloy band theory.

One can rewrite the susceptibility integral equation (2.6) in the matrix form

$$
\chi(\omega)=\bar{\chi}(\omega)+\bar{\chi}(\omega) \delta \mathbf{u}(\omega) \chi(\omega),
$$

where the matrix elements $\bar{\chi}_{i j}(\omega)$ are determined by Fourier transformation from $\bar{\chi}(\overrightarrow{\mathbf{q}}, \omega)$, which is given by Eq. (3.14). In analogy with the $t$ matrix for $G_{i j}(\omega)$, one can define the $t^{\chi}$ matrix for $\chi_{i j}(\omega)$,

$$
\mathbf{t}^{\chi}(\omega) \equiv \delta \mathbf{u}(\omega)+\delta \mathbf{u}(\omega) \bar{\chi}(\omega) \mathbf{t}^{\chi}(\omega),
$$

in terms of which Eq. (3.18) assumes the form

$$
\chi(\omega)=\bar{\chi}(\omega)+\bar{\chi}(\omega) \mathbf{t}^{\chi}(\omega) \bar{\chi}(\omega) .
$$

Upon inverting Eq. (3.19), we find 


$$
\begin{aligned}
\mathbf{t}^{\chi}(\omega) & =\delta \mathbf{u}(\omega)[1-\bar{\chi}(\omega) \delta \mathbf{u}(\omega)]^{-1} \\
& \equiv \delta \mathbf{u}(\omega) \mathbf{A}^{-1}(\omega)
\end{aligned}
$$

and substituting this expression into Eq. (3.20), we obtain the final result,

$$
\chi(\omega)=\bar{\chi}(\omega)+\bar{\chi}(\omega) \delta \mathbf{u}(\omega) \mathbf{A}^{-1}(\omega) \bar{\chi}(\omega),
$$

which is identical to Eq. (2.11) which was found in Sec. II by FT techniques.

\section{Renormalized-interactor and $T$-matrix formalisms for $\chi_{i j}(\omega)$ for the case of off-diagonal disorder}

In this subsection, we present a generalization to the case of off-diagonal disorder of the alloy band formalisms developed in Secs. III B and III C for the case of diagonal disorder. In the geometric approximation, discussed in Sec. II B, the method of Shiba ${ }^{9}$ is easily applied to the calculation of $\chi_{i j}(\omega)$, yielding exactly the same results, Eqs. (2.20) and (2.21), as were obtained in Sec. II B. Thus, we confine our discussion in this section to the more general case in which the matrix elements of the bare alloy band susceptibility, $\Gamma_{i j}(\omega)$, depend only on the chemical occupation of sites $i$ and $j$, but in which the geometric approximation does not hold. In that case, the use of the formalism of alloy band theory allows one to obtain a new, possibly superior, solution of the equation of motion (3.3) for $\chi(\omega)$. In this subsection we use the alloy band theory of Blackman, Esterling, and Berk ${ }^{5}$ (BEB) for binary alloys, $A_{1-c} B_{c}$, as reexpressed by Gonis and Garland ${ }^{6}$ to derive an alternative to Eq. (2.28) in which the matrix to be inverted is a $2 n \times 2 n$ matrix rather than an $\left(n+n^{\prime}\right) \times\left(n+n^{\prime}\right)$ matrix. As is shown in Appendix B, the generalization of this theory to multicomponent alloys is straightforward.

Blackman et al. showed that in alloy band theory the off-diagonal disorder associated with randomness in the transfer matrix elements, $\boldsymbol{W}_{i j}$, can be transformed into diagonal disorder by the introduction of $2 \times 2$ matrices provided that the $W_{i j}$ depend only on the chemical occupation of sites $i$ and $j$. Upon introducing the configuration-space matrices (we consider explicitly the case of binary alloys),

$$
\begin{aligned}
& \underline{G}_{i j}(\omega) \equiv\left(\begin{array}{ll}
x_{i} G_{i j}(\omega) x_{j} & x_{i} G_{i j}(\omega) y_{j} \\
y_{i} G_{i j}(\omega) x_{j} & y_{i} G_{i j}(\omega) y_{j}
\end{array}\right), \\
& \underline{g}_{i}(\omega) \equiv\left(\begin{array}{cc}
x_{i} g^{A}(\omega) & 0 \\
0 & y_{i} g^{B}(\omega)
\end{array}\right),
\end{aligned}
$$

and

$$
\underline{W}_{i j} \equiv\left(\begin{array}{ll}
W_{i j}^{A A} & W_{i j}^{A B} \\
W_{i j}^{B A} & W_{i j}^{B B}
\end{array}\right),
$$

where the projection operator, $x_{i} \equiv 1-y_{i}$, is equal to 1 if the site $i$ is occupied by an atom of type $A$ and is zero otherwise, one finds that the equation of motion (3.1) assumes the form

$$
\left.\underline{G}_{i j}(\omega)=\underline{g}_{i}(\omega) \mid \delta_{i j} \underline{1}+\sum_{k} \underline{W}_{i k} \underline{G}_{k j}(\omega)\right\}
$$

in site representation or

$$
\underline{\mathbf{G}}(\omega)=\mathbf{g}(\omega)[\underline{1}+\underline{\mathbf{W}} \mathbf{G}(\omega)]
$$

in supermatrix form. Here and in the following, a single underline denotes a $2 \times 2$ matrix in configuration space, whereas boldface type together with an underline denotes a matrix in site representation whose matrix elements are BEB $2 \times 2$ matrices. Note that the matrices $\underline{W}_{i j}$ are translationally invariant, so that Eq. (3.26) is formally analogous to the equation for the alloy Green's function in the presence of only diagonal disorder, with the only disorder arising from the projection operators in $\mathbf{g}_{i}(\omega)$.

This formalism cannot be applied directly to the integral equation (3.3) for $\chi(\omega)$ because the matrix, $\Gamma(\omega)$, which carries the off-diagonal disorder does not always occur sandwiched between two diagonal matrices. However, we show here how it can be used with either the renormalized-interactor formalism of Sec. III B or the $t$ matrix formalism of Sec. IIIC. We consider the renormalized-interactor approach. The BEB formalism can be directly applied to Eq. (3.4) of Sec. III B, which is exactly analogous to Eq. (3.1) of alloy band theory. Upon introducing the $2 \times 2$ matrix generalizations, $\underline{\underline{g}}_{i j}(\omega), \underline{q}_{i}(\omega)$, and $\underline{\mathcal{W}}_{i j}(\omega)$, of the scalars $\mathcal{S}_{i j}(\omega), \mathscr{g}_{i}(\omega)$, and $\mathcal{W}_{i j}(\omega)$ in analogy with the definitions (3.23)-(3.25), one can rewrite Eq. (3.4) in the form

$$
\underline{\mathcal{Q}}(\omega)=\boldsymbol{q}^{(\omega)}[\underline{1}+\underline{\mathcal{W}}(\omega) \underline{\mathcal{Q}}(\omega)] .
$$

Here, the site-diagonal matrix $\mathscr{Z}_{i i}(\omega)$ is given by the equation

$$
\begin{aligned}
\underline{q}_{i i}(\omega) & \equiv\left[\underline{1}-\underline{u}_{i} \underline{\gamma}(\omega)\right]^{-1} \\
& =\underline{1}+\underline{u}_{i} \underline{\gamma}(\omega) \underline{q}_{i}(\omega),
\end{aligned}
$$

where

$$
\underline{u}_{i} \equiv\left(\begin{array}{cc}
x_{i} u^{A} & 0 \\
0 & y_{i} u^{B}
\end{array}\right)
$$

and

$$
\underline{\gamma}(\omega) \equiv\left(\begin{array}{cc}
\gamma^{A}(\omega) & 0 \\
0 & \gamma^{B}(\omega)
\end{array}\right) .
$$

Note that the matrices $\underline{\mathcal{W}}_{i j}(\omega)$ and $\underline{\gamma}(\omega)$ are now translationally invariant. Thus, for the case of a cluster embedded in an effective medium, one can define the corresponding matrices, $\overline{\mathcal{W}}_{i j}(\omega)$ and $\underline{\underline{\gamma}}(\omega)$, for the effective medium to be equal to $\underline{\mathcal{W}}_{i j}$ and $\underline{\gamma}$, respectively. Any self-consistency conditions on the effective medium can always be satisfied by an appropriate choice of $\overline{\mathbf{u}}(\omega)$ provided that one allows $\underline{\mathbf{u}}(\omega)$ to be only cluster diagonal, rather than site diagonal. The only disorder remaining in Eq. (3.27) is the diagonal disorder associated with $q_{i}(\omega)$.

Upon generalizing the renormalized-interactor formalism of Sec. III B, Eq. (3.9a) assumes the form

$$
\underline{\mathcal{g}}_{i i}(\omega)=\underline{q}_{i}(\omega)\left[\underline{1}+\underline{u}_{i} \underline{\Delta}_{i}(\omega) \underline{\underline{S}}_{i i}(\omega)\right],
$$

or, equivalently, 


$$
\underline{g}_{i i}(\omega)=\left[\underline{1}-\mathscr{g}_{i}(\omega) \underline{u}_{i} \underline{\Delta}_{i}(\omega)\right]^{-1} \mathscr{g}_{i}(\omega),
$$

where the fully renormalized interactor is defined by the equation

$$
\underline{\Delta}_{i}(\omega) \equiv\left(\begin{array}{cc}
\Delta_{i}^{A A}(\omega) & \Delta_{i}^{A B}(\omega) \\
\Delta_{i}^{B A}(\omega) & \Delta_{i}^{B B}(\omega)
\end{array}\right) .
$$

In order to solve Eq. (3.31b) we follow the procedure of Sec. III B, embedding the site (or cluster) 0 in an effective medium characterized by an effective renormalized interactor, $\bar{\Delta}(\omega)$, given by the expression

$$
\bar{\Delta}(\omega)=\underline{\bar{u}}^{-1}(\omega)\left[\bar{q}^{-1}(\omega)-\underline{\bar{g}}_{00}(\omega)^{-1}\right],
$$

where $\underline{\underline{u}}(\omega), \bar{q}(\omega)$, and $\underline{\mathscr{S}}_{00}(\omega)$ are the $2 \times 2$ matrix generalizations of the scalars $\bar{u}(\omega), \bar{g}(\omega)$, and $\overline{\mathscr{S}}_{00}(\omega)$, with $\bar{g}(\omega)$ and $\bar{S}_{00}(\omega)$ defined by Eqs. (3.12)-(3.14). Note that the matrix $\overline{\mathbf{u}}(\omega)$ now can have off-diagonal matrix elements in both site space and configuration space, in contrast with Sec. III B, although it remains cluster diagonal.

Upon substituting Eqs. (3.33) and (3.28) into Eq. (3.31b) and inverting the resultant equation, one finds the expression

$$
\begin{aligned}
\underline{\underline{g}}_{00}(\omega)^{-1}= & {\left[\underline{1}-\underline{u}_{0} \underline{\gamma}(\omega)\right] } \\
& \times\left\{\underline{1}-\underline{q}_{0}(\omega) \underline{u}_{0} \underline{\bar{u}}^{-1}(\omega)\right. \\
& \left.\times\left[\bar{q}^{-1}(\omega)-\underline{\bar{g}}_{00}(\omega)^{-1}\right]\right\} .
\end{aligned}
$$

After performing some straightforward algebra and reinverting this equation, one obtains the result

$$
\underline{\mathcal{g}}_{00}(\omega)=\underline{\bar{g}}_{00}\left[1-\delta \underline{u}_{0}(\omega) \underline{\bar{\chi}}_{00}(\omega)\right]^{-1},
$$

where $\bar{\chi}_{00}(\omega)$ is the $2 \times 2$ matrix generalization of the scalar $\bar{\chi}_{00}(\omega)$. Then, expressing $\underline{\mathcal{Q}}_{00}(\omega)$ in terms of $\underline{\chi}_{00}(\omega)$, one finds the result

$$
\underline{\chi}_{00}(\omega)=\left[1-\underline{\chi}_{00}(\omega) \delta \underline{u}_{0}(\omega)\right]^{-1} \underline{\bar{\chi}}_{00}(\omega),
$$

which is equivalent to Eq. (3.17) of Sec. III B for the case of diagonal disorder. However, Eq. (3.36) is defined in a different space than is Eq. (3.17), with a $2 n \times 2 n$ rather than an $n \times n$ matrix to be inverted. The physical susceptibility, $\chi_{00}(\omega)$, is given by the equation

$$
\chi_{00}(\omega)=\sum_{\alpha=1}^{2} \sum_{\beta=1}^{2}\left[\underline{\chi}_{00}(\omega)\right]_{\alpha \beta},
$$

and the physical susceptibility of the effective medium is given by

$$
\bar{\chi}_{i j}(\omega)=\sum_{\alpha=1}^{2} \sum_{\beta=1}^{2}\left[\underline{\bar{\chi}}_{i j}(\omega)\right]_{\alpha \beta} .
$$

Finally, upon generalizing the $t$-matrix formalism for $\chi(\omega)$ presented in Sec. III C by introducing $2 \times 2$ matrices, as was done within the renormalized-interactor formalism, one can rewrite Eq. (3.20) in the form

$$
\underline{\chi}(\omega)=\underline{\bar{\chi}}(\omega)+\underline{\bar{\chi}}(\omega) \underline{t}^{\chi}(\omega) \underline{\bar{\chi}}(\omega) .
$$

Because scattering occurs only on the single site (or clus- ter) $i$, which is embedded in the effective medium, one may write

$$
\underline{\chi}_{00}(\omega)=\underline{\bar{\chi}}_{00}(\omega)+\underline{\bar{\chi}}_{00}(\omega) \underline{t}^{\chi}(\omega) \underline{\bar{\chi}}_{00}(\omega),
$$

where $\underline{t}^{\chi} \equiv \underline{t}_{00}^{\chi}$. By substituting Eq. (3.36) for $\underline{\chi}_{00}(\omega)$ into Eq. (3.40), one finds

$$
\begin{aligned}
\underline{t}^{\chi}(\omega) & =\delta \underline{u}_{0}(\omega)\left[\underline{1}-\underline{\chi}_{00}(\omega) \delta \underline{u}_{0}(\omega)\right]^{-1} \\
& \equiv \delta \underline{u}_{0}(\omega) \underline{A}_{0}^{-1}(\omega) .
\end{aligned}
$$

Now, upon substituting Eq. (3.41) into Eq. (3.39), one finds the result,

$\underline{\chi}_{i j}(\omega)=\underline{\bar{\chi}}_{i j}(\omega)+\underline{\bar{\chi}}_{i 0}(\omega) \delta \underline{u}_{0}(\omega) \underline{A}_{0}^{-1}(\omega) \underline{\chi}_{0 j}(\omega)$,

which is analogous to Eq. (2.11) of Sec. II A for the case of diagonal disorder. However, the matrix $\underline{A}_{0}(\omega)$ to be inverted here is a $2 n \times 2 n$ matrix, rather than an $n \times n$ matrix. Note that Eq. (3.42) gives the susceptibility $\chi_{i j}(\omega)$ for any two sites (or clusters) $i$ and $j$ in the crystal, whereas the more restricted susceptibility equation (3.36) gives the local susceptibility only within the embedded cluster, 0. Note also that Eqs. (3.41) and (3.42) could be obtained directly by generalizing Eqs. (3.18) and (3.19) of Sec. III C because our introduction of $2 \times 2$ matrices has transformed the off-diagonal disorder in $\underline{\Gamma}(\omega)$ into diagonal form, leaving only a disorder in $\underline{\mathbf{u}}(\omega)$. Finally, the physical uniform static susceptibility, $\chi(0)$, of the alloy is given by the equation

$$
\begin{aligned}
\chi(0) & =N^{-1} \sum_{i, j} \sum_{\alpha=1}^{2} \sum_{\beta=1}^{2}\left[\underline{\chi}_{i j}(0)\right]_{\alpha \beta} \\
& =\bar{\chi}(0)+N^{-1} \sum_{\alpha=1}^{2} \sum_{\beta=1}^{2}\left[\underline{\chi}(0) \delta \underline{u}_{0}(0) \underline{A}_{0}^{-1}(0) \underline{\bar{\chi}}(0)\right]_{\alpha \beta},
\end{aligned}
$$

where

$$
\bar{\chi}(0) \equiv N^{-1} \sum_{i, j} \bar{\chi}_{i j}(0) \text { and } \underline{\bar{\chi}}(0)=N^{-1} \sum_{i, j} \underline{\bar{\chi}}_{i j}(0) .
$$

\section{SELF-CONSISTENT DETERMINATION OF THE EFFECTIVE MEDIUM}

In this section we discuss, and examine briefly, different possible self-consistency conditions for determining the effective medium in which a site or cluster is to be embedded. The equations presented here are given for the case of diagonal disorder only, but can be immediately applied to the case of off-diagonal disorder by simply adding an underline under every quantity in each equation, i.e., by replacing each matrix, $\mathbf{M}$, by the corresponding supermatrix, $\underline{\mathbf{M}}$. As was shown in detail in Sec. III C, the case of off-diagonal disorder becomes exactly analogous to the case of purely diagonal disorder upon introducing the appropriate supermatrix generalizations.

In a single-site theory one considers the case of a single site embedded in a translationally invariant effective medium characterized by the effective intra-atomic Coulomb potential, $\bar{u}(\omega)$. In such a theory one can in- 
troduce a formalism equivalent to either the locator (or renormalized interactor) or the propagator (or $t$-matrix) formalism employed in the single-site CPA (SSCPA) of alloy band theory, as has been shown in Sec. III. The self-consistency conditions which correspond to those of the SSCPA band theory are immediately obvious. In such a theory of the susceptibility, $\bar{u}(\omega)$ can be determined either by the locator condition,

$$
\left\langle\chi_{00}(\omega)\right\rangle_{\mathrm{ss}}=\bar{\chi}_{00}(\omega) \text {, }
$$

or by the propagator condition, ${ }^{10}$

$$
\left\langle t^{\chi}(\omega)\right\rangle_{\mathrm{SS}}=0 \text {, }
$$

where the symbol, $\langle\cdots\rangle_{\text {ss }}$, denotes an ensemble average over the occupation of a single site. It is obvious from Eq. (3.20) that the conditions (4.1) and (4.2) are exactly equivalent, just as the locator and propagator selfconsistency conditions are exactly equivalent within the SSCPA in alloy band theory. Furthermore, by proofs analogous to those of alloy band theory, these conditions give rise to an analytic effective potential, $\bar{u}(\omega)$, even for the case of off-diagonal disorder, and give analytic local susceptibilities, $\bar{\chi}_{i j}(\omega)$, in the absence of long-range magnetic order.

The exact ensemble-average alloy susceptibility over all alloy configurations can be expressed in the general form

$$
\langle\chi(\omega)\rangle=\bar{\chi}(\omega)+\bar{\chi}(\omega)\left\langle\mathbf{T}^{\chi}(\omega)\right\rangle \bar{\chi}(\omega),
$$

where $\mathbf{T}^{\chi}(\omega)$, the total $T$ matrix for the alloy susceptibility, describes the scattering due to the difference between the intra-atomic Coulomb potential of the real, disordered material and the effective potential, $\overline{\mathbf{u}}(\omega)$, of the effective medium. Upon expanding the total $\mathbf{T}^{\chi}$ matrix in terms of the single-site $t^{\chi}$ matrices, one can rewrite Eq. (4.3) in site representation in the form

$$
\begin{array}{r}
\left\langle\chi_{i j}(\omega)\right\rangle=\bar{\chi}_{i j}(\omega)+\sum_{k} \bar{\chi}_{i k}(\omega)\left\langle t_{k}^{\chi}(\omega)\right\rangle \bar{\chi}_{k j}(\omega) \\
+\sum_{k, l \neq k} \bar{\chi}_{i k}(\omega)\left\langle t_{k}^{\chi}(\omega) \bar{\chi}_{k l}(\omega)\right. \\
\left.\times t_{l}^{\chi}(\omega)\right\rangle \bar{\chi}_{l j}(\omega)+\cdots .
\end{array}
$$

Then, imposing the condition (4.2), it is obvious from Eq. (4.4) that $\left\langle\chi_{i j}(\omega)\right\rangle$ is equal to $\bar{\chi}_{i j}(\omega)$, correct up to terms of order $\left\langle\left(t_{k}^{\chi} t_{l}^{\chi}\right)^{2}\right\rangle$. Thus, the SSCPA provides the best single-site approximation to the alloy susceptibility, just as it provides the best single-site approximation to the single-particle alloy Green's functions, $G_{i j}(\omega)$.

In cluster theory one considers the case of a compact $n$-site cluster embedded in an effective medium, which may either be determined self-consistently or be chosen in some other way. Unfortunately, the problem of choosing the proper effective medium into which to embed an $n$-site cluster of real atoms is much more critical in determining the ensemble-averaged uniform susceptibility of an alloy than in determining an alloy density of states. One reason for the criticality is that $\chi(\omega)$ for the alloy cannot be calculated even in principle solely from the elements, $\left\langle\chi_{i j}(\omega)\right\rangle$, of the ensemble-averaged susceptibility matrix for the embedded cluster, whereas the alloy density of states $n(\omega)$ is in principle equal to the spectral-weight function $A_{i}(\omega)$ for any site in the embedded cluster. Thus, $\chi(\omega)$ depends explicitly on the embedding effective medium, whereas $n(\omega)$ depends on it only to the extent that it affects the spectral weight $A_{0}(\omega)$ on the central site of the embedded cluster. Second, for strongly-exchange-enhanced alloys the dependence of $\chi_{i i}(\omega)$ on the local environment of the site $i$ is much more nonlinear than is the dependence of $A_{i}(\omega)$ on local environment. Third, and probably most important, unlike the dependence of $A_{i}(\omega)$ on local environment, the dependence of $\chi_{i i}(\omega)$ on local environment is very long ranged for strongly-exchange-enhanced alloys.

Because one must consider large clusters $(n \gtrsim 55)$ in any accurate calculation of the susceptibility of strongly-exchange-enhanced alloys, for simplicity we consider here only site-diagonal cluster theories, within which the intra-atomic Coulomb potential, $\bar{u}(\omega)$, of the effective medium is chosen to be not only cluster diagonal but also site diagonal. We consider three different self-consistent site-diagonal cluster theories which are analogous to existing cluster band theories: the selfconsistent central-site approximation (SCCSA), ${ }^{11}$ the self-consistent boundary-site approximation (SCBSA), ${ }^{12}$ and the $t$-matrix self-consistent central-site approximation (TMSCCSA). Within the SCCSA one imposes the single scalar condition

$$
\left\langle\chi_{00}(\omega)\right\rangle_{C}=\bar{\chi}_{00}(\omega) \text {, }
$$

where 0 denotes the central site of the cluster and the symbol $\langle\cdots\rangle_{C}$ denotes an average over all configurations of the cluster. On the other hand, within the SCBSA one replaces the condition (4.5) by the condition

$$
\left\langle\chi_{n n}(\omega)\right\rangle_{C}=\bar{\chi}_{00}(\omega),
$$

where $n$ denotes a boundary site in the cluster, with the boundary defined in some convenient way. Finally, in the TMSCCSA one imposes the single scalar condition,

$$
\left\langle t_{00}^{\chi}\right\rangle_{C}=0 \text {, }
$$

where 0 denotes the central site of the cluster $C$, and $t^{\chi}$ is the cluster $t$ matrix. It can be shown ${ }^{13}$ that the TMSCCSA always yields analytic results and thus is a theory at least in principle superior to the SCCSA and the SCBSA.

In addition to considering the three site-diagonal selfconsistency conditions discussed above, Eqs. (4.5)-(4.7), we also consider here the self-consistency condition

$$
\langle\chi(\omega)\rangle_{C}=\bar{\chi}(\omega),
$$

or, equivalently,

$$
N^{-1} \sum_{i, j}\left\langle\chi_{i j}(\omega)\right\rangle_{C}=\bar{\chi}(\omega)
$$

where the sum over $i$ and $j$ runs over all sites and is not limited to the cluster $C$. This new self-consistency condition, along with the conditions $(4.5)-(4.7)$, reduces the 
case of only diagonal disorder to the usual SSCPA selfconsistency conditions $(4.1)$ and (4.2) in the limit of a single site, as is obvious from Eqs. (2.11) and (3.42). Also, for the case of geometric off-diagonal disorder, the condition (4.8) is very nearly equivalent to the conditions (4.1) and (4.2), as is shown by Eqs. (2.26) and (2.27). Further, this condition has two apparent advantages over any of the site-diagonal conditions, Eqs. (4.5)-(4.7). First, it is a condition directly on the quantity $\bar{\chi}(\omega)$, which one wishes to calculate. Second, because $\bar{\chi}(\omega)$ depends much more sensitively on $\bar{u}$ than does $\bar{\chi}_{00}(\omega)$, Eqs. (4.8) should determine $\bar{u}$ much more sensitively than do any of Eqs. (4.5)-(4.7).

However, there exist two other criteria for choosing the best self-consistency condition. Both of those criteria favor the TMSCCSA condition, Eq. (4.7). First is the criterion of analyticity. Of the cluster-theory selfconsistency conditions, Eqs. (4.5)-(4.8), only Eq. (4.7) satisfies that criterion. Second is the numerical criterion of rapid convergence toward the final correct value of $\chi(\omega)$ with increasing cluster size. It is apparent that the value of $\bar{\chi}(\omega)$ should approach its final value most rapidly with increasing cluster size for the case in which $\bar{\chi}(\omega)$ [or $\bar{u}(\omega)$ ] is determined primarily in terms of quantities evaluated near the center of the embedded cluster; with increasing cluster size those quantities must converge to their final values more rapidly than the same quantities evaluated near the edge of the cluster. One can immediately discard the SCBSA, condition (4.6), on the basis of this criterion. Also, this criterion argues against the use of the condition (4.8). Of the two remaining conditions, the SCCSA condition and the TMSCCSA condition, both are favored by this criterion because both are expressed in terms of the ensemble average of a matrix element evaluated at the cluster center. In order to compare these two conditions in terms of this criterion, we express the SCCSA condition, Eq. (4.5), in the form

$$
\begin{aligned}
\left\langle\chi_{00}(\omega)\right\rangle_{C}- & \bar{\chi}_{00}(\omega) \\
& =\left[\bar{\chi}(\omega)\left\langle\delta \mathbf{u}(\omega) \mathbf{A}^{-1}(\omega)\right\rangle_{C} \bar{\chi}(\omega)\right]_{00}=0
\end{aligned}
$$

and the TMSCCA condition in the form

$$
\left\langle t_{00}^{\chi}(\omega)\right\rangle=\left[\left\langle\delta \mathbf{u}(\omega) \mathbf{A}^{-1}(\omega)\right\rangle_{C}\right]_{00}=0,
$$

where all matrices are defined only over the space of the cluster $C$ and where the matrix $\mathbf{A}(\omega)$ is defined by Eq. (2.10). It is clear from Eqs. (2.10), (4.9), and (4.10) that for fixed $\bar{u}(\omega)$, the quantity $\left\langle t_{00}^{\chi}(\omega)\right\rangle_{C}$ converges to its final value more rapidly with increasing cluster size than does $\left\langle\chi_{00}(\omega)\right\rangle_{C}$. This conclusion is supported by the results of model numerical calculations.

We conclude that of the three site-diagonal selfconsistency conditions considered, the TMSCCA condition is the best from all points of view. We also have found that the TMSCCA self-consistency condition is definitely preferable to the condition (4.8) with respect to the criteria of analyticity and of rapidity of convergence with increasing cluster size. We believe that on balance these arguments for use of the TMSCCA condition, Eq. (4.10), outweigh the arguments for the use of the condition (4.8).

Having suggested a best method for the determination of a self-consistent cluster-CPA effective medium, we must issue a caveat to the reader. Unlike the case for alloy band calculations, the averaging over clusters containing as many as 55 or more atoms embedded in an effective medium can lead to large errors in the calculation of the exchange-enhanced susceptibility. This is especially true for very-highly-exchange-enhanced hosts, such as $\mathrm{Pd}$, near the critical concentration for ferromagnetism. In the worst case, that of $\mathrm{Pd}-\mathrm{Ni}$ alloys, for 55-site clusters embedded in a self-consistent effective medium it leads to an overestimate of the susceptibility by a factor of order 2 at the critical concentration. This difficulty arises from the extremely nonlinear dependence of the local susceptibilities $\chi_{i j}(\omega)$ on the local environments of the sites $i$ and $j$, coupled with the extremely long range of $\chi_{i j}$ in such alloys. One obtains substantially better values for the susceptibility of $\mathrm{Pd}-\mathrm{Ni}$ alloys near the critical concentration by considering successively larger numbers of $\mathrm{Ni}$ atoms embedded in pure $\mathrm{Pd}$ than by performing a cluster-CPA calculation. On the other hand, for alloys such as $\mathrm{Ni}-\mathrm{Cu}$, for which the critical concentration is not very close to either one or zero, the cluster CPA appears to be the best technique for the calculation of the susceptibility. The different methods of actually performing calculations are discussed in detail and illustrative actual results are given in paper IV of this series.

\section{DISCUSSION AND CONCLUSIONS}

The formalism presented in this paper represents a substantial advance over all previous theories for the calculation of the exchange-enhanced magnetic susceptibility of substitutionally disordered alloys. Furthermore, this paper is unique in that it establishes a link between the formalism of alloy band theory and that of alloy susceptibility. With the exception of the phenomenological Landau theory of Kato and Mathon, ${ }^{8}$ all such previous theories can be obtained from the formalism presented here by making the appropriate approximations. By considering only the case of isolated single impurities in an exchange-enhanced host one arrives at the theory of Lederer and Mills $^{2}$ or Engelsberg et al. ${ }^{14}$ By considering the case of single atoms embedded in a selfconsistently-determined CPA effective medium one can arrive at the results of Harris and Zuckermann, ${ }^{10} \mathrm{Kato}$ and Shimizu, ${ }^{15}$ Hasegawa and Kanamori, ${ }^{16}$ Levin et al. ${ }^{17}$ Fukuyama, ${ }^{18}$ Inoue and Shimizu, ${ }^{19}$ or, in principle, those of Hirooka and Shimizu. ${ }^{20}$ By virtue of the very nature of single-site averaging, none of those theories can incorporate local-environment effects, which we have found numerically to be very important, or can correctly incorporate the effects of short-range order. In particular, those theories yield a qualitatively wrong concentration dependence of the static uniform susceptibility, $\chi(0)$. These problems can be overcome by using the full cluster formalism presented in this paper. It is important to realize that approximations to the method presented here can easily destroy its integrity and usefulness; the previous cluster theories of Brouers et $a l,{ }^{21}$ and of Van der Rest, ${ }^{22}$ which represent approximations to the present formalism, failed to include exchange enhancement in the effective medium or to treat exactly 
the embedding of a cluster within a given effective medium.

The theory of Kato and Mathon is the only previous theory of the exchange-enhanced magnetic susceptibility of alloys not to be included, at least in principle, in the present formalism. That phenomenological theory did go beyond the present paper in several respects, but gave unphysical results which could be corrected only by abandoning self-consistency and introducing extra parameters. However, by combining the results of papers II and III in this series of papers with the formalism of this paper, one can regain all of the advantages of the results of Kato and Mathon without sacrificing the advantages of a self-consistent, more nearly first-principles formalism. Furthermore, our numerical results, to be presented in paper IV of this series, are qualitatively superior to those of Kato and Mathon.

The present theory is written in a local or Wannier representation, which may suggest its use with a tightbinding or interpolation-scheme Hamiltonian. But, it can be used with values of the $\Gamma_{i j}$ obtained by Fourier transforming the results of any band calculation of the $\Gamma^{m m^{\prime}}(\overrightarrow{\mathbf{q}})$ for the different components of the alloy to be studied. The intra-atomic Coulomb interactions $u_{i}$ for the different components of the alloy can be determined from self-consistent local-density theory ${ }^{23}$ using standard band-theory calculational methods for pure elements. More accurate values of the $u_{i}$ could be calculated using self-consistent alloy band-structure techniques ${ }^{24}$ or by correcting the values for the pure elements using an approximation such as the $t$-matrix approximation of Hirooka and Shimizu. ${ }^{20}$ Thus, the present formalism in principle can be used for a purely first-principles calculation of $\chi(\overrightarrow{\mathbf{q}})$ for any substitutionally disordered alloy in the absence or neglect of local moment formation.

On the other hand, the values of $u_{i}$ and of the $\Gamma_{i j}$ for one or more of the components of an alloy can be treated as variable parameters or can be determined by experiments on the alloy components. For example, $u_{i}$ and the $\Gamma_{i j}$ for pure $\mathrm{Pd}$ can be estimated from the bandcalculated value for $\Gamma(0)$, the experimental value for $\chi(0)$, and the range, $\lambda$, of the magnetic polarization clouds induced in pure $\mathrm{Pd}$ by isolated $\mathrm{Fe}$ impurities. Calculations show that different sets of values for the $\Gamma_{i j}$ consistent with the same values for $\Gamma(0)$ and $\lambda$ yield essentially indistinguishable results for the static susceptibilities, $\chi_{i j}(0)$, for $\mathrm{Pd}-\mathrm{Ni}$ alloys. ${ }^{25}$ In practice, for a highly-exchange-enhanced alloy one must leave one parameter adjustable in order to obtain good agreement with experiment for $\chi(0)$. Usually, it is most convenient to allow this free parameter to be the Coulomb interaction $u_{i}$ of that alloy component for which $u_{i}$ is the largest. That is because the value $\chi(0)$ for such an alloy typically depends very strongly on that value of $u_{i}$; for example, in $\mathrm{Pd}-\mathrm{Ni}$ alloy a $0.1 \%$ variation in the value of $u_{i}$ on $\mathrm{Ni}$ atoms produces a change in $\chi(0)$ as large as $20 \% .^{26}$

This paper does leave several major problems unresolved. First, the use of the RPA in this paper precludes the treatment of spin-fluctuation effects and, hence, leads to an overestimate of $\chi(0)$ at all concentra- tions for any alloy system. For alloy systems which possess a critical concentration for ferromagnetism, that overestimate is serious near that concentration; for such alloy systems, $\chi(0)$ approaches infinity at all concentrations as the size of the clusters treated approaches infinity. Also, the temperature dependence of the susceptibility is not treated in this paper. Both of these problems are resolved, at least in major part, in the third paper of this series, in which the effects of quantum and thermal spin fluctuations are introduced in a manner which yields results consistent with those $e^{1}$ of renormalization-group theory for the case of an isolated impurity.

Second, the formalism of this paper does not allow one to calculate the susceptibilities $\chi_{i j}(\omega)$ in the presence of induced magnetization or spontaneous local moments. Thus, it is directly applicable only to alloy systems, such as $\mathrm{Rh}-\mathrm{Pd}$, in which no local moments are formed and only to the calculation of the low-field susceptibility. However, this problem is resolved in paper II of this series. In that paper a new formalism is introduced for the calculation of induced and spontaneous local moments and magnetizations, and the formalism of this paper is extended so as to allow the calculation of the susceptibilities $\chi_{i j}(\omega)$ in the presence of known local moments.

Two remaining problems have not been treated either in the existing literature or in this series of papers. The first is the effect of internal thermal spin fluctuations in magnetic clusters or spin-polarization clouds, which are here assumed to be ferromagnetically aligned internally. That assumption leads to an overestimate of both the magnetization and the susceptibility at elevated temperatures. The second is the effect of random interactions between magnetic clusters or spin-polarization clouds, which can induce spin-glass behavior. This effect has been totally ignored in this series of papers and in all other papers not based on the Heisenberg model. However, it is in principle amenable to treatment using an extension of the formalism presented in this series of papers.

\section{ACKNOWLEDGMENTS}

The authors would like to acknowledge many useful discussions with Dr. J. S. Kouvel. This work was partially supported by the U.S. Department of Energy under Contract No. W-7405-ENG-48 with Lawrence Livermore National Laboratory.

\section{APPENDIX A}

In this appendix we give a detailed derivation of Eq. (1.2). Neglecting the effects associated with the $s-d$ hybridization between the $d$ bands and conduction bands and interatomic Coulomb and exchange interactions, the electronic structure of an alloy can be described by the degenerate Hubbard Hamiltonian, 


$$
\begin{aligned}
H= & \sum_{\substack{i, j, \sigma \\
m, m^{\prime}}} t_{i j}^{m m^{\prime}} c_{i m \sigma}^{\dagger} c_{j m^{\prime} \sigma}+\sum_{i, m, \sigma} \varepsilon_{i m} n_{i m \sigma}+\sum_{i, m, m^{\prime}} U_{i}^{m m^{\prime}} n_{i m \uparrow} n_{i m^{\prime} \downarrow} \\
& +\frac{1}{2} \sum_{\substack{i, \sigma, m, m^{\prime} \\
\left(m^{\prime} \neq m\right)}}\left(U_{i}^{m m^{\prime}}-J_{i}^{m m^{\prime}}\right) n_{i m \sigma^{\prime}} n_{i m^{\prime} \sigma}-\left(g \mu_{B} / 2\right) \sum_{i, m} H_{i m}\left(n_{i m \uparrow}-n_{i m \downarrow}\right) e^{i \omega t}
\end{aligned}
$$

where $i$ and $j$ are site indices and $m$ and $m^{\prime}$ are band indices. The transfer terms, $t_{i j}^{m m^{\prime}}$, describe the hopping of electrons from band $m^{\prime}$ on site $j$, to band $m$ on site $i$, and can in general depend on the band indices $m$ and $m^{\prime}$, the relative displacement and chemical occupation of sites $i$ and $j$, and their local environment. The sitediagonal electronic energies, $\varepsilon_{i m}$, and the intra-atomic Coulomb and exchange interactions, $U_{i}^{m m^{\prime}}$ and $J_{i}^{m m^{\prime}}$, depend on the chemical occupation of site $i$, its local environment and the band indices $m$ and $m^{\prime}$. The last term represents the Zeeman energy resulting from a time- and space-dependent external magnetic field applied parallel to the $z$ axis.

As was recognized by Caroli et al. ${ }^{27}$ and by Dworin and Narath ${ }^{28}$ the Hamiltonian in Eq. (A1) is not invariant under rotation in spin and coordinate space. However, the terms necessary to restore these symmetries do not affect the physics of the problem and, hence, are neglected here.

The expectation value of the $z$ component of the induced magnetic moment of the orbital $m$ on site $i$ in the presence of the field is equal to

$$
\left\langle\mu_{i m}\right\rangle \equiv g \mu_{B}\left\langle S_{i m}\right\rangle \equiv\left(g \mu_{B} / 2\right)\left\langle n_{i m \uparrow}-n_{i m \downarrow}\right\rangle,
$$

Here, $\left\langle n_{i m \sigma}\right\rangle$ is given by

$$
\left\langle n_{i m \sigma}\right\rangle=\frac{1}{2} \bar{n}_{i m}+\operatorname{sgn}\{\sigma\}\left\langle S_{i m}\right\rangle,
$$

where $\bar{n}_{i m}$ is the time-independent average value of $n_{i m}$ in the absence of a field and where $\left\langle S_{i m}\right\rangle$ vanishes in the absence of an applied field. The enhanced (or interacting) local susceptibility, $\chi_{i j}^{m m^{\prime}}(\omega)$, represents the linear response of the alloy when electron-electron interactions are taken into account and is defined by

$$
\begin{aligned}
\chi_{i j}^{m m^{\prime}}(\omega) & \equiv d\left\langle\mu_{i m}\right\rangle / d H_{j m^{\prime}} e^{-i \omega t} \\
& \equiv 2 d\left\langle S_{i m}\right\rangle / d h_{i m^{\prime}} e^{-i \omega t},
\end{aligned}
$$

where $H_{j m^{\prime}}=g \mu_{B} h_{j m^{\prime}}$ and where the units $\left(g \mu_{B}\right)^{2}=2$ have been taken for convenience.

However, in the simplest approximation, the effects of exchange interactions can be investigated within a generalized molecular field approximation which is equivalent to a time-dependent Hartree-Fock (HF) (or RPA) approximation. In this approximation, any product of number operators $n_{i m \sigma} n_{i m^{\prime} \sigma^{\prime}}$ in the Hamiltonian (A1) is replaced by the corresponding single-particle time-independent operator,

$$
\left\langle n_{i m \sigma}\right\rangle n_{i m^{\prime} \sigma^{\prime}}+n_{i m \sigma}\left\langle n_{i m^{\prime} \sigma^{\prime}}\right\rangle .
$$

Making this approximation in the Hamiltonian (A1), one obtains the HF (or RPA) expression

$$
\begin{aligned}
H^{\mathrm{HF}}= & \sum_{\substack{i, j, \sigma \\
m, m^{\prime}}} t_{i j}^{m m^{\prime}} c_{i m \sigma}^{\dagger} c_{i m^{\prime} \sigma}+\sum_{i, m, \sigma} V_{i m}^{\mathrm{HF}} n_{i m \sigma} \\
& -\frac{1}{2} \sum_{i, m} h_{i m}^{\mathrm{eff}}\left(n_{i m \uparrow}-n_{i m \downarrow}\right) e^{i \omega t}
\end{aligned}
$$

where

$$
\begin{aligned}
V_{i m}^{\mathrm{HF}} & =\varepsilon_{i m}+\frac{1}{2}\left(U_{i}^{m m} \bar{n}_{i m}+\sum_{m^{\prime} \neq m}\left(2 U_{i}^{m m^{\prime}}-J_{i}^{m m^{\prime}}\right) \bar{n}_{i m^{\prime}}\right) \\
& \equiv \varepsilon_{i m}+\frac{1}{2} \sum_{m^{\prime}} v_{i}^{m m^{\prime}} \bar{n}_{i m^{\prime}}
\end{aligned}
$$

and

$$
\begin{aligned}
h_{i m}^{\mathrm{eff}} & =h_{i m}+2\left(U_{i}^{m m}\left\langle S_{i m}\right\rangle+\sum_{m^{\prime} \neq m} J_{i}^{m m^{\prime}}\left\langle S_{i m^{\prime}}\right\rangle\right] e^{-i \omega t} \\
& \equiv h_{i m}+2 \sum_{m^{\prime}} u_{i}^{m m^{\prime}}\left\langle S_{i m^{\prime}}\right\rangle e^{-i \omega t},
\end{aligned}
$$

with

$$
v_{i}^{m m^{\prime}}=\left(2-\delta_{m m^{\prime}}\right) U_{i}^{m m^{\prime}}-\left(1-\delta_{m m^{\prime}}\right) J_{i}^{m m^{\prime}}
$$

and

$$
u_{i}^{m m^{\prime}}=\delta_{m m^{\prime}} U_{i}^{m m^{\prime}}+\left(1-\delta_{m m^{\prime}}\right) J_{i}^{m m^{\prime}} .
$$

This HF Hamiltonian describes a multiple band of electrons moving in the effective potential, $V^{\mathrm{HF}}$, and subject to the spatially nonuniform effective magnetic field, $h^{\text {eff }}$, which gives rise to an inhomogeneous magnetization. The first two terms in this Hamiltonian give the alloy band structure, namely, the local spectral weight functions,

$$
A_{i j \sigma}^{m m^{\prime}}(\omega)=-\pi^{-1} \operatorname{Im}\left[G_{i j \sigma}^{m m^{\prime}}(\omega)\right],
$$

the partial densities of states,

$$
n_{\sigma}^{m}(\omega)=N^{-1} \sum_{i} A_{i i \sigma}^{m m}(\omega)
$$

and the total density of states,

$$
n \sigma(\omega)=N^{-1} \sum_{i, m}, \quad A_{i i \sigma}^{m m}(\omega)=\sum_{m} n_{\sigma}^{m}(\omega),
$$

etc., where $N$ is the number of atoms in the crystal, and hence determine the local unenhanced (band) susceptibilities, $\Gamma_{i j}^{m m^{\prime}}$, which are determined from the Hamiltonian (A5) with the effective field, $h_{i m}^{\text {eff }}$, replaced by the applied field, $h_{i m}$. The last term in Eq. (A7) for the effective field, $h_{i m}^{\text {eff }}$, just gives rise to the enhancement of the local susceptibility, $\chi_{i j}^{m m^{\prime}}$.

The expectation value of the spin, $\left\langle S_{\mathrm{im}}\right\rangle$, thus can be expressed either in terms of the applied fields, 


$$
\left\langle S_{i m}\right\rangle=\frac{1}{2} \sum_{j, m^{\prime}} \chi_{i j}^{m m^{\prime}}(\omega) h_{i m^{\prime}} e^{i \omega t},
$$

or in terms of the effective fields,

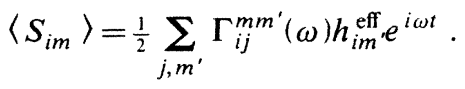

Inserting the expression (A10a) for $\left\langle S_{i m}\right\rangle$ into Eq. (A7) for $h_{j m}^{\text {eff }}$, substituting the resultant expression into Eq. (A10b), and then equating Eq. (A10a) and (A10b), one obtains the integral equation

$$
\chi_{i j}^{m m^{\prime}}(\omega)=\Gamma_{i j}^{m m^{\prime}}(\omega)+\sum_{k, m^{\prime \prime}, m^{\prime \prime \prime}} \Gamma_{i k}^{m m^{\prime \prime}}(\omega) u_{k}^{m^{\prime \prime} m^{\prime \prime \prime}} \chi_{k j}^{m^{\prime \prime \prime} m^{\prime}}(\omega) .
$$

Equation (A11) is the starting equation, Eq. (1.2), for the derivation of the results presented in this paper. It is clear that all of the results of this paper carry over to the case of a nondegenerate band upon replacing $u_{k}$ by $U_{k}$ everywhere in the paper and that they carry over to the case of the most general $(2 l+1)$-fold band upon replacing all scalar quantities in the paper by the corresponding $(21+1) \times(2 l+1)$ matrices.

\section{APPENDIX B}

In this appendix we generalize the binary alloy theory of Sec. III to multicomponent alloys with general ODD. First we consider the generalization of the bandtheoretic results, Eqs. (3.23)-(3.26), and then we show how this generalization applies to the calculation of the susceptibility of $m$-component alloys with disorder in the $\Gamma_{i j}$.

We consider a system consisting of $m$ kinds of atoms, $A_{1} A_{2}, \ldots, A_{m}$, distributed in some fashion over the $N$ sites of a regular lattice with corresponding probabilities $c_{1}, c_{2}, \ldots, c_{m}$. Clearly, the concentrations $c_{\alpha}$ satisfy the sum rule

$$
\sum_{\alpha=1}^{m} c_{\alpha}=1
$$

The site-diagonal elements of the alloy Hamiltonian, $\varepsilon_{i}$, can assume any one of the values, $\varepsilon_{A_{1}}, \varepsilon_{A_{2}}, \ldots, \varepsilon_{A_{m}}$, in correspondence with the kind of atom occupying site $i$, and the off-diagonal elements $W_{i j}$ can assume the values

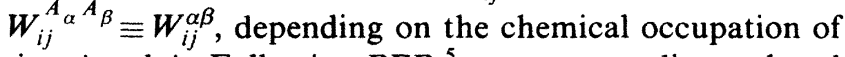
sites $i$ and $j$. Following BEB, ${ }^{5}$ we separate diagonal and off-diagonal disorder through the introduction of projection operators $x_{i \alpha}, \alpha=1,2, \ldots, m$, which satisfy the relations

$$
x_{i \alpha} x_{i \beta}=x_{i \alpha} \delta_{\alpha \beta}
$$

and

$$
\sum_{\alpha=1}^{m} x_{i \alpha}=1
$$

for any site $i$ in the lattice. In the $m$-component configuration space defined by the $x_{i \alpha}$, the appropriately generalized forms of the $2 \times 2$ matrices given in Eqs. (3.23) $-(3.25)$ are given by the expressions

$\underline{G}_{i j} \equiv\left(\begin{array}{cccc}x_{i_{1}} G_{i j} x_{j_{1}} & x_{i_{1}} G_{i j} x_{j_{2}} & \cdots & x_{i_{1}} G_{i j} x_{j_{m}} \\ x_{i_{2}} G_{i j} x_{j_{1}} & x_{i_{2}} G_{i j} x_{j_{2}} & \cdots & x_{i_{2}} G_{i j} x_{j_{m}} \\ \vdots & \vdots & & \vdots \\ x_{i_{m}} G_{i j} x_{j_{1}} & x_{i_{m}} G_{i j} x_{j_{2}} & \cdots & x_{i_{m}} G_{i j} x_{j_{m}}\end{array}\right)$,

$\underline{g}_{i} \equiv\left(\begin{array}{cccc}x_{i_{1}} g^{A_{1}} & 0 & \cdots & 0 \\ 0 & x_{i_{2}} g^{A_{2}} & \cdots & 0 \\ \vdots & \vdots & & \vdots \\ 0 & 0 & \cdots & x_{i_{m}} g^{A_{m}}\end{array}\right)$

and

$$
\underline{W}_{i j} \equiv\left(\begin{array}{cccc}
\boldsymbol{W}_{i j}^{11} & W_{i j}^{12} & \cdots & \boldsymbol{W}_{i j}^{1 m} \\
\boldsymbol{W}_{i j}^{21} & W_{i j}^{22} & \cdots & \boldsymbol{W}_{i j}^{2 m} \\
\vdots & \vdots & & \vdots \\
\boldsymbol{W}_{i j}^{m 1} & \boldsymbol{W}_{i j}^{m 2} & \cdots & \boldsymbol{W}_{i j}^{m m}
\end{array}\right) .
$$

With these definitions, the equations of motion for the Green's function, Eqs. (3.26a) and (3.26b), remain valid. Only the interpretation of the matrix elements of the supermatrices in Eqs. (3.26b) and (3.27) is to be changed from $2 \times 2$ to $m \times m$ matrices. The entire discussion following Eq. (3.26) now carries through in terms of $m \times m$ matrix elements. In particular, upon introducing the $m \times m$ matrices

$$
\begin{aligned}
& \underline{u}_{i}=\left(\begin{array}{ccccc}
x_{i_{1}} u^{A_{1}} & 0 & 0 & \cdots & 0 \\
0 & x_{i_{2}} u^{A_{2}} & 0 & \cdots & 0 \\
\vdots & \vdots & & & \vdots \\
0 & 0 & & \cdots & x_{i_{m}} u^{A_{m}}
\end{array}\right), \\
& \underline{\gamma}=\left(\begin{array}{ccccc}
\gamma^{A_{1}} & 0 & 0 & \cdots & 0 \\
0 & \gamma^{A_{2}} & 0 & \cdots & 0 \\
\vdots & \vdots & \vdots & & \vdots \\
0 & 0 & 0 & \cdots & \gamma^{A_{m}}
\end{array}\right)
\end{aligned}
$$

and

$$
\underline{\Delta}_{i}=\left(\begin{array}{cccc}
\Delta_{i}^{11} & \Delta_{i}^{12} & \cdots & \Delta_{i}^{1 m} \\
\Delta_{i}^{21} & \Delta_{i}^{22} & \cdots & \Delta_{i}^{2 m} \\
\vdots & \vdots & & \vdots \\
\Delta_{i}^{m 1} & \Delta_{i}^{m 2} & \cdots & \Delta_{i}^{m m}
\end{array}\right)
$$


and noting that the sums in the equations corresponding to Eqs. (3.37) and (3.38) should extend to $m$ rather than to 2, one easily derives Eq. (3.42) in terms of supermatrices of $m \times m$ matrices. In particular, in place of Eq. (3.43) one easily obtains the following expression for the uniform static susceptibility of the alloy,

$$
\begin{aligned}
\chi(0)= & N^{-1} \sum_{i, j} \sum_{\alpha=1}^{m} \sum_{\beta=1}^{m}\left[\chi_{i j}(0)\right]_{\alpha \beta} \\
=\bar{\chi}(0)+N^{-1} \sum_{\alpha=1}^{m} \sum_{\beta=1}^{m}[ & \bar{\chi}(0) \underline{\delta u}_{0}(0) \\
& \left.\times \underline{A}_{0}^{-1}(0) \underline{\bar{\chi}}(0)\right]_{\alpha \beta} .
\end{aligned}
$$

*Present address: Department of Physics, California State University at Northridge, Northridge, CA 91330.

${ }^{1}$ H. R. Krishna-Murthy, K. G. Wilson, and J. W. Wilkins, Phys. Rev. Lett. 35, 1101 (1975); Phys. Rev. B 21, 1003 (1980).

${ }^{2}$ P. Lederer and D. L. Mills, Solid State Commun. 5, 131 (1967); Phys. Rev. 165, 837 (1968).

${ }^{3}$ A. Gonis and J. W. Garland, Phys. Rev. B 16, 2424 (1977); 18, 3999 (1978).

${ }^{4}$ P. Soven, Phys. Rev. B 156, 809 (1967); 178, 1136 (1969); R. J. Elliott, J. A. Krumhansl and P. L. Leath, Rev. Mod. Phys. 46, 465 (1974); J. S. Faulkner, in Progress in Materials Science, edited by J. W. Christian, P. Hassen, and T. B. Massalski (Pergamon, New York, 1982), Nos. 1 and 2.

5J. A. Blackman, D. M. Esterling, and N. F. Berk, Phys. Rev. B 4, 2412 (1971).

${ }^{6}$ A. Gonis and J. W. Garland, Phys. Rev. B 16, 1495 (1977).

${ }^{7}$ There are strong indications that in many, if not all, alloys of magnetic and nonmagnetic species spin-glass order precedes ferromagnetism as the concentration of the magnetic species is increased. In such metallic alloys, there may be no critical concentation for ferromagnetism in the strictest sense; however, such considerations are outside the purview of this paper, in which spin-glass order is not considered.

${ }^{8}$ T. Kato and J. Mathon, J. Phys. F 6, 221 (1976); 1341 (1976).

${ }^{9}$ H. Shiba, Prog. Theor. Phys. 46, 77 (1971).

${ }^{10}$ R. Harris and M. J. Zuckermann, Phys. Rev. B 5, 101 (1972).

${ }^{11}$ F. Brouers, F. Ducastelle, F. Gautier and J. Van der Rest, J. Phys. F 3, 2120 (1973).

${ }^{12}$ W. H. Butler, Phys. Rev. B 15, 5267 (1977).

${ }^{13}$ F. Ducastelle, J. Phys. C 7, 1975 (1974).
${ }^{14}$ S. Engelsberg, W. F. Brinkman, and S. Doniach, Phys. Rev. Lett. 20, 1040 (1968).

${ }^{15}$ T. Kato and M. Shimizu, J. Phys. Soc. Jpn. 33, 363 (1972).

${ }^{16}$ H. Hasegawa and J. Kanamori, J. Phys. Soc. Jpn. 31, 382 (1971); 33, 1599 (1972); 33, 1607 (1972).

${ }^{17}$ K. Levin, R. Bass, and K. H. Bennemann, Phys. Rev. B 6, 1865 (1972).

${ }^{18}$ H. Fukuyama, Phys. Rev. B 5, 2872 (1972); in Magnetism and Magnetic Materials 1972 (Denver), Proceedings of the 18th Annual Conference on Magnetism and Magnetic Materials, AIP Conf. Proc. No. 10, edited by C. D. Graham and J. J. Rhyne (AIP, New York, 1973).

${ }^{19}$ J. Inoue and M. Shimizu, J. Phys. Soc. Jpn. 40, 1321 (1976); 42, 1547 (1977); Phys. Lett. 60A, 45 (1977).

${ }^{20}$ S. Hirooka and M. Shimizu, J. Phys. Soc. Jpn. 43, 70 (1977).

${ }^{21}$ F. Brouers, F. Gautier, and J. Van der Rest. J. Phys. F 5, 975 (1975).

22J. Van der Rest, J. Phys. F 7, 1051 (1977); 8, 1263 (1978).

${ }^{23}$ V. von Barth and L. Hedin, J. Phys. C 5, 1629 (1972); O. Gunnarsson and B. I. Lundqvist, Phys. Rev. B 13, 4274 (1976).

${ }^{24}$ G. W. Stocks and H. Winter, Z. Phys.. B 46, 95 (1982); Phys. Rev. B 27, 882 (1983).

${ }^{25}$ J. W. Garland and N. Kioussis, J. Appl. Phys. 55, 2356 (1984).

${ }^{26}$ N. Kioussis, J. W. Garland, and J. S. Kouvel (unpublished).

${ }^{27}$ B. Caroli, P. Lederer, and D. Saint-James, Phys. Rev. Lett. 23, 700 (1969); B. Caroli, C. Caroli, and D. R. Fredkin, Phys. Rev. 178, 599 (1969).

${ }^{28}$ L. Dworin and A. Narath, Phys. Rev. Lett. 25, 1287 (1970). 